\title{
Mathematical models for blood flow in elastic vessels: Theory and numerical analysis
}

by

Qian Li

\author{
A thesis \\ submitted to the Victoria University of Wellington \\ in fulfilment of the \\ requirements for the degree of \\ Master \\ in Mathematics.
}

Victoria University of Wellington

2019 



\begin{abstract}
In this thesis we study model equations that describe the propagation of pulsatile flow in elastic vessels. Since dealing with the Navier-Stokes equations is a very difficult task, we derive new asymptotic weakly non-linear and weakly-dispersive Boussinesq systems. Properties of the these systems, such as the well-posedness, and existence of travelling waves are being explored. Finally, we discretize some of the new model equations using finite difference methods and we demonstrate their applicability to blood flow problems. First we introduce the basic equations that describe fluid flow in elastic vessels and previously derived systems. We also review previously derived model equations for fluid flow in elastic tubes. We start with the description of the equations of motion of elastic vessel. Then we derive asymptotically Boussinesq systems for fluid flow in elastic vessels. Because these systems are weakly non-linear and weakly dispersive we expect then to have solitary waves as special solutions. We explore some possibilities by construction analytical solutions. After that we continue the derivation of the previous chapter. We derive a general system where the horizontal velocity is evaluated at any distance from the center of the tube. Special emphasis is paid on the case of constant radius vessels. We also derive unidirectional models and obtain the dissipative Boussinesq system by taking the viscosity effects into account. There is also an alternative derivation of the general system when considering the equations of potential flow. We show that the two different derivations lead to the same system. The alternative derivation is based on asymptotic series expansions. Then we develop finite difference methods for the numerical solution of the BBM equation and for the classical Boussinesq system studied in the previous chapters. Finally, we demonstrate the application of the new models to blood flow problems. By performing several numerical
\end{abstract}


simulations. 


\section{Contents}

1 Introduction 1

2 Model equations for fluid flow in elastic vessels 5

2.1 Equation of vessel wall . . . . . . . . . . . . . . 5

2.2 The Euler equations . . . . . . . . . . . . . . . . . 9

2.3 Approximate models for fluid flow in vessels . . . . . . . . . . . . 12

2.4 A 1-D Boussinesq system . . . . . . . . . . . . . . . . 16

3 Asymptotic derivation of Boussinesq systems 19

3.1 Boussnesq model . . . . . . . . . . . . . . . . 19

3.2 The equations at the center of the vessel . . . . . . . . 25

3.3 Solitary waves of the Boussnesq system $\ldots \ldots \ldots 27$

4 A general system $\quad 29$

4.1 Further developments . . . . . . . . . . . . . . . . . 29

4.2 The system with constant radius . . . . . . . . . . 32

4.3 One way propagation models . . . . . . . . . . . . 34

4.4 Dissipative Boussinesq systems . . . . . . . . . . . . 37

5 Properties of the general system $\quad 41$

5.1 Dispersion relation for the Euler equations . . . . . . . . 41

5.2 Dispersion relation of the Boussinesq system . . . . . . . . 44

5.3 Linear well-posedness of the Boussinesq system . . . . . . . 46

5.4 Well-possedness of the non-linear system . . . . . . . . 51

5.5 Solitary waves of the general system . . . . . . . . . 58 
5.6 Existence of solitary waves . . . . . . . . . . . . 60

5.7 Symmetries ........................ 63

6 Alternative derivation of the Boussinesq system 65

6.1 Derivation of the additional system . . . . . . . . . . . . . 65

6.2 The general system . . . . . . . . . . . . . . . . 69

6.3 Viscoelastic correction . . . . . . . . . . . . 71

7 Numerical Methods $\quad 73$

7.1 Finite difference method . . . . . . . . . . . . 73

7.2 A finite difference method for the BBM equation . . . . . . 74

7.3 Stability analysis of the numerical method for the BBM equa-

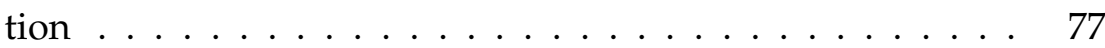

7.4 A numerical method for the Boussinesq system . . . . . . . 78

7.5 Numerical experiments . . . . . . . . . . . . . . . . . 79

8 Conclusions $\quad 85$ 


\section{Chapter 1}

\section{Introduction}

The cardiovascular system consists of the heart, the arteries and the veins. The arteries carry oxygenated blood to the organs. So the arteries are very important for the physiological and pathological studies. Many cardiovascular diseases are related to arteries. The large and medium arteries can be approximated by elastic tubes with cylinderical symmetry.

In 1775, Euler derived a system of partical differnetial equations, which is now used to describle flows without viscosity [10]. For this system of equations it is almost impossible to find analytical solutions. Then Young found that the motion of flood flow can be seen as "wave", [24]. The wave nature of the blood flow makes it convenient to compute the "Frenquency" of the blood flow.

In physiological conditions, the Euler equations are essentially weakly nonlinear thus it is very helpful to study the linearized system [22]. In 1877, the Moens-Korteweg equation for the wave speed has been derived, [2] [15]. Witzig is the first author to publish the results on wave propagation in elastic vessels [21].

In 1957, Womersley derived a two-dimensional system which analyse the flow in the frequency domain [22]. However, the frequency domain analysis assumes that the arterial system is in a state of oscillation, which is not true in reality. In the duration of diastole and systole, the behaviour of the blood flow is totally different, and the frenquency analysis cannot distinguish the diffenrence. 
It is usually very difficult to study the motion of the blood flow in the vessel [13] when considering viscotiy. The Navier-Stokes equations are second order non-linear partial differentail equations, which makes the study very difficult. For simplification, many reduced models have been derived to study the blood flow in vessels. One-dimensional models have been widely used, [11] [19]. One-dimensional model was first used by Euler [10]. The increasing computational ability of computers make numerical methods very convenient. It has been showed that one-dimensioanl models in the time domain rather than the frenquency domain are good descriptions of the non-linear flow [1] [14].

But most of these studies are based on the constant radius and average velocity along the radius. Additionally, some simplifications imposed in the one dimensional models make the mmodel lose some other informations. In our study we consider a vessel with variables radius, which expands our knowledge scope of the blood flow.

In this thesis, we derive asymptotic models for blood flow in elastic vessels with cylinderical symmetry and variable radius. Our system is derived from the Euler equations [5]. We will obtain a Boussinesq system which couple the horizontal velocity of the fluid $u$ and the displacement of the the vessel wall $\eta$.

In the study of the shallow water waves, Boussinesq systems are widely used, [4]. However, our system is not totally the same as that of the shallow water waves. Some new properties and characteristics have been found. We can obtain further unidirectional model equations, the result of which can be compared with that of the Boussinesq systems.

The method of potential flow is also used in the study of water waves, [8]. Now we apply it in our derivations too. Also, we can obtain higher order systems by iteration with the potential flow method.

This thesis has the following structure: In Chapter 2, we present the basic equations we used and then a review of previously derived models. In Chapter 3, we derived the new systems which are of Boussinesq type. In Chapter 4 , we extend the derivation of Chapter 3 and obtain general systems by introducing a parameter $\theta$ and evaluating the horizontal velocities 
at different radii. We also consider the viscous correction in this chapter. In Chapter 5, we study some properties of the general systems such as dispersion relations and well-posedness of the systems. In Chapter 6, we apply an alternative derivation, which is essentially based on perturbation series expansions. We again obatin the same results as in the previous chapters. In Chapter 7, we study numerically the effects of some radius variations to blood flow. demonstrating the useful of the new models.

Since we have derived the new system of the blood flow, we believe that it will be helpful to improve the study of physiology and pathology related to arteries. We also extend the range of application of the Boussinesq system and find some interesting results of the new system. Using this study, further results can be induced and other system related to fluid flow in visco-elastic pipes can be derived, while other models can also be justified using asymptotic reasoning.

Most of the results of this study have been published. 


\section{Chapter 2}

\section{Model equations for fluid flow in elastic vessels}

In this chapter we introduce the basic equations that describe fluid flow in elastic vessels and previously derived systems. We also review previously derived model equations for fluid flow in elastic tubes. We start with the description of the equations of motion of elastic vessel.

\subsection{Equation of vessel wall}

Denote an element of the vessel wall by $V$. Let its thickness be $h=\delta r$ and the arc length be $r_{0} \delta \theta$, where $r_{0}$ is the radius of the tube. Let the axial length of the tube be $\delta x$ and denote the density of the tube wall by $\rho_{w}$. Thus we have the volume $\delta V$ of this element, and the mass $\delta m$ of this element:

$$
\delta V \approx h r_{0} \delta \theta \delta x, \quad \delta m \approx \rho_{w} \delta V .
$$

Here we take the average density of the vessel.

$$
\delta m \approx \rho_{w} \delta V .
$$

Then, there exist four mechanical stresses that exerted on the tube wall and all of them with the dimensions of force per unit area. 


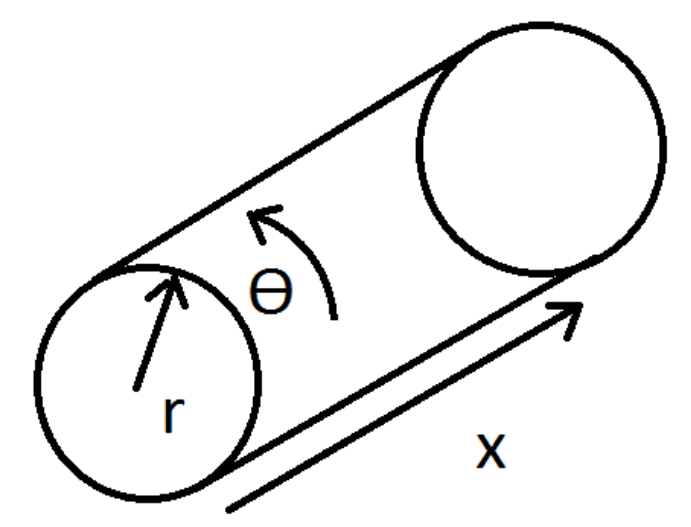

Figure 2.1: Coordinates for the vessel

(i) Denote the tension on the axial direction by $S_{x x}$, which leads to a force in the positive $x$ direction due to a change $\delta S_{x x}$ over the length of the element. It is given as below:

$$
\delta S_{x x} \cdot h r_{0} \delta \theta=\frac{\partial S_{x x}}{\partial x} \delta x h r_{0} \delta \theta
$$

(ii) Denote the radial stress by $S_{r r}$, which is related to the angular tension within the vessel wall. Then there exist a force that is parallel to the radius of the tube and it is toward the center of the section:

$$
-S_{r r} \cdot r_{0} \delta \theta \delta x
$$

(iii) The net difference between the pressures that are inside and outside the vessel, $p_{w}$, produces a force that is outward in the radius direction. The force is given as:

$$
p_{w} \cdot r_{0} \delta \theta \delta x
$$

(iv) Shear stress $\tau_{w}$ that acting by the blood inside the vessel lead to a force 

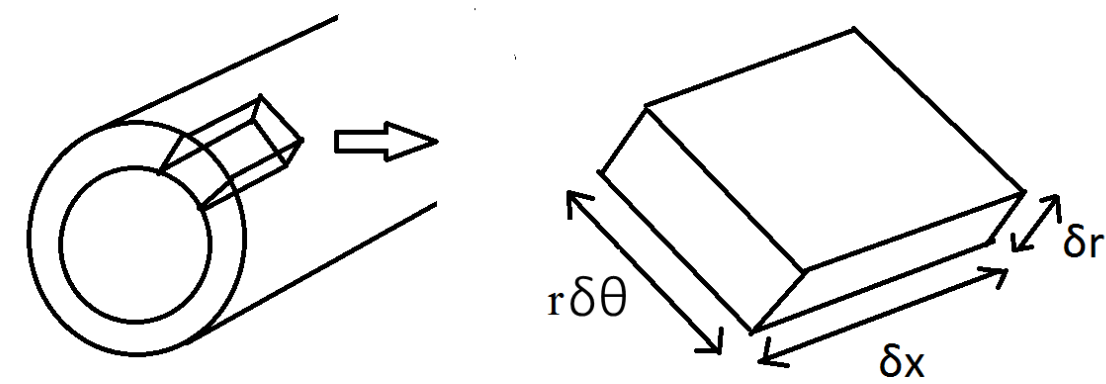

Figure 2.2: Element of the vessel wall

in the blood fluid direction, which is given as:

$$
\tau_{w} \cdot r_{0} \delta \theta \delta x
$$

Denote the displacement in the $x, r$ and $\theta$ direction by $\xi, \eta$ and $\phi$. Consider the Newton's second law of motion, we write the equations in the axial direction as:

$$
\rho_{w} \cdot h r_{0} \delta \theta \frac{d^{2} \xi}{d t^{2}}=h r_{0} \delta \theta \cdot \frac{\partial S_{x x}}{\partial x} \delta x+r_{0} \delta \theta \delta x \cdot \tau_{w} .
$$

Dividing both sides of this equation by $r_{0} \delta \theta \delta x$, we have

$$
\rho_{w} h \frac{d^{2} \xi}{d t^{2}}=h \frac{\partial S_{x x}}{\partial x}+\tau_{w} .
$$

In the radial direction we have

$$
\rho_{w} \cdot h r_{0} \delta \theta \delta x \cdot \frac{d^{2} \eta}{d t^{2}}=r_{0} \delta \theta \delta x \cdot p_{w}-r_{0} \delta \theta \delta x \cdot S_{r r}
$$

Similarly, we can simplify it to

$$
\rho_{w} h \cdot \frac{d^{2} \eta}{d t^{2}}=p_{w}-S_{r r}
$$

Since the external forces in the angular direction is zero, considering 
the axial symmetry we have that the angular direction acceleration is zero. However, there is a relation between the angular and the radical strain, [25].

$$
S_{r r}=\frac{h}{r_{0}} S_{\theta \theta}
$$

By the stress-strain relations, we can express $S_{r r}, S_{x x}$ and $S_{\theta \theta}$ in terms of $\eta$ and $\xi$. Denote the strains in the axial radial and angular directions by $e_{x x}, e_{r r}$ and $e_{\theta \theta}$, and let $\mathrm{E}$ be Young's modulus and $\sigma$ be Poisson's ratio, then the strain-stress relations for an elastic body are given by:

$$
\begin{aligned}
& e_{x x}=\frac{1}{E}\left[S_{x x}-\sigma\left(S_{r r}+S_{\theta \theta}\right)\right], \\
& e_{r r}=\frac{1}{E}\left[S_{r r}-\sigma\left(S_{\theta \theta}+S_{x x}\right)\right], \\
& e_{\theta \theta}=\frac{1}{E}\left[S_{\theta \theta}-\sigma\left(S_{r r}+S_{x x}\right)\right] .
\end{aligned}
$$

Assuming that $r_{0} / h$ is much larger than $\sigma$, and with Eq.(2.9), we have that

$$
e_{x x}=\frac{1}{E}\left[S_{x x}-\frac{\sigma r_{0}}{h} S_{r r}\right]
$$

and

$$
e_{\theta \theta}=\frac{1}{E}\left[\frac{r_{0}}{h} S_{r r}-\sigma S_{x x}\right] .
$$

Let $E_{\sigma}=\frac{E}{1-\sigma^{2}}$, then solving for $S_{x x}$ and $S_{r r}$, we have

$$
S_{x x}=E_{\sigma}\left(e_{x x}+\sigma e_{\theta \theta}\right)
$$

and

$$
S_{r r}=\frac{h E_{\sigma}}{r_{0}}\left(e_{\theta \theta}+\sigma e_{x x}\right) .
$$

Considering $\xi$ as a function of $x$, then for a small element of the wall of length $\delta x$, we have

$$
\delta x+\delta \xi=\delta x+\frac{\partial \xi}{\partial x} \delta x
$$

Thus

$$
e_{x x}=\frac{\partial \xi}{\partial x}
$$


Then we know that axial stain can be defined as the ratio of the change in length over the original length.

The angular strain is mainly from the radial displacement $\eta$, so for the angular strain we have that

$$
e_{\theta \theta}=\frac{1}{r_{0} \delta \theta}\left[\left(r_{0}+\eta\right) \delta \theta-r_{0} \delta \theta\right]=\frac{\eta}{r_{0}} .
$$

Combining Eq.(2.6), Eq.(2.8), Eq.(2.15) and Eq.(2.16), we have the equations of motion of the tube wall

$$
\begin{aligned}
& \frac{\partial^{2} \xi}{\partial t^{2}}=\frac{E_{\sigma}}{\rho_{w}}\left(\frac{\partial^{2} \xi}{\partial x^{2}}+\frac{\sigma}{r_{0}} \frac{\partial \eta}{\partial x}\right)-\frac{\tau_{w}}{\rho_{w} h}, \\
& \frac{\partial^{2} \eta}{\partial t^{2}}=\frac{p_{w}}{\rho_{w} h}-\frac{E_{\sigma}}{\rho_{w} r_{0}}\left(\frac{\eta}{r_{0}}+\sigma \frac{\partial \xi}{\partial x}\right) .
\end{aligned}
$$

The second derivative on the left side of Eq.(2.21) is very small. Since the element we studied seldom moves in direction of $x, \frac{\partial \xi}{\partial x}$ can also be neglected. Then we obtain the simplified relation:

$$
\frac{p_{w}}{\rho_{w} h}=\frac{E_{\sigma}}{\rho_{w} r_{0}} \frac{\eta}{r_{0}}
$$

We continue with the description of the equation of fluid motion in an elastic vessel.

\subsection{The Euler equations}

We first assume that the fluid in the vessel is incompressible and inviscid. For convenience, we also assume that the flood flow is axisymmetric, which means that the velocity in the direction that is perpendicular to the radius is 0 , and this direction is usually denoted by $\theta$ in cylinderical coordinates system, as well as the acceleration in this direction. So we only consider the axial velocity $\bar{v}$, which is along the radius and the horizontal velocity $\bar{u}$, which is along the axis of the vessel. Then the Eular equations of the fluid motion can be written in cylinderical coordinates are: 


$$
\begin{gathered}
\bar{u}_{\bar{t}}+\overline{u u}_{\bar{x}}+\overline{v u}_{\bar{r}}+\frac{1}{\rho} \bar{p}_{\bar{x}}=0, \\
\bar{v}_{\bar{t}}+\overline{u v}_{\bar{x}}+\overline{v v}_{\bar{r}}+\frac{1}{\rho} \bar{p}_{\bar{r}}=0, \\
\bar{u}_{\bar{x}}+\bar{v}_{\bar{r}}+\frac{1}{\bar{r}} \bar{v}=0 .
\end{gathered}
$$

Denoting the radius of the vessel wall by $\bar{r}^{w}$, then obviously we have $\bar{r}^{w}=\bar{r}_{0}+\bar{\eta}$, where $\bar{\eta}$ is the displacement of the vessel wall and $\bar{r}_{0}$ is the radius of the vessel in resting position. Here we only consider the displacement in the radius direction. It is noted that, $r_{0}$ is only a function of $x$. By the no-slip condition [5], we know that

$$
\left.\bar{v}\right|_{\bar{r}=\bar{r}^{w}}=\frac{d}{d \bar{t}} \bar{r}^{w}=\bar{\eta}_{\bar{t}}+\left.\left(\overline{r_{0}}+\bar{\eta}\right)_{x} \bar{u}\right|_{\bar{r}=\bar{r}^{w}}
$$

We thereby have that for $\bar{r}=\bar{r}^{w}$,

$$
\bar{v}=\bar{\eta}_{\bar{t}}+\left(\bar{r}_{0 \bar{x}}+\bar{\eta}_{\bar{x}}\right) \bar{u},
$$

which is the first boundary condition. We also have that [25]:

$$
\rho^{w} h \bar{\eta}_{\overline{t t}}=\bar{p}^{w}-\frac{\bar{E}_{\sigma}(\bar{x}) h}{\bar{r}_{0}^{2}} \bar{\eta} .
$$

This equation is from Eq.(2.21) after droping the term for $\xi$. In the above equation, $\rho^{w}$ is the density of the vessel wall and $h$ is the thickness of the vessel wall. $\bar{p}^{w}$ denotes the pressure at the vessel wall for the fluid. Let $\bar{E}$ be the Young modulus and $\sigma$ be the Poisson ratio, then $\bar{E}_{\sigma}=\frac{\bar{E}}{1-\sigma^{2}}$. Now we have the second boundary condition. Additionally, we have $\left.\bar{v}\right|_{\bar{r}=0}=$ 0 because the motion is axisymmetric. Now we can do a scaling for the variables. We can make the following changes of variables:

$$
\bar{x}=\bar{\Lambda} x, \quad \bar{r}=\bar{R} r, \quad \bar{\eta}=\bar{A} \eta, \quad \bar{t}=\frac{\bar{\Lambda}}{\bar{c}} t .
$$

In the above equations, $\bar{\Lambda}$ is a typical wave length of the waves, $\bar{R}$ is a typical radius, and $\bar{A}$ is a typical amplitude of the wall displacement. The $\bar{c}$ 
is the Moens-Korteweg velocity of a wave propagating without nonlinear terms. $\bar{c}$ has the form:

$$
\bar{c}=\sqrt{\frac{\bar{E} h}{2 \bar{R} \rho} .}
$$

where $\rho$ is the density of the fluid. Then we take

$$
\epsilon=\frac{\bar{A}}{\bar{R}}, \delta=\frac{\bar{R}}{\bar{\Lambda}}
$$

We thereby use the long-wave and small amplitude :

$$
\epsilon \ll 1, \delta^{2}=k \epsilon, k=O(1)
$$

The velocities can then be scaled as:

$$
\bar{u}=\epsilon c u, \bar{v}=\epsilon c \delta v .
$$

We also take $\bar{p}=\epsilon \rho c^{2} p$.

Let $\alpha=\frac{\rho_{w} h}{\rho R}$ and $\beta(x)=\frac{2 E(x)}{r_{0}^{2}(x)}$, we have the following six equations in non-dimensional and scaled form:

$$
\begin{array}{r}
u_{t}+\epsilon u u_{x}+\epsilon v u_{r}+p_{x}=0, \\
\delta^{2}\left[v_{t}+\epsilon u v_{x}+\epsilon v v_{r}\right]+p_{r}=0, \\
r u_{x}+(r v)_{r}=0, \\
v\left(r^{w}\right)=\eta_{t}+r_{x}^{w} u, \\
\alpha \delta^{2} \eta_{t t}=p^{w}-2 \beta(x) \eta, \\
v\left(r^{w}\right)=\eta_{t}+r_{0 x} u\left(r^{w}\right)+\epsilon \eta_{x} u\left(r^{w}\right) .
\end{array}
$$

$E(x)=\frac{\bar{E}_{\sigma}(x)}{\bar{E}}$ here is a non-dimensional quantity: 


\subsection{Approximate models for fluid flow in vessels}

In this section we review the derivation of a unidirectional model due to Cascaval [5]. We define two new variables independent to each other:

$$
\xi=\epsilon x, \quad \tau=G(x ; \epsilon)-t .
$$

If we take $g=\frac{d G}{d x}$, we can transform the partial derivatives as below

$$
\begin{gathered}
\partial_{t}=\frac{\partial}{\partial \tau} \frac{\partial \tau}{\partial t}=-\partial_{\tau} \\
\partial_{x}=\frac{\partial \xi}{\partial x} \frac{\partial}{\partial \xi}+\frac{\partial \tau}{\partial x} \frac{\partial}{\partial \tau}=\epsilon \partial_{\xi}+g(\xi) \partial_{\tau}
\end{gathered}
$$

Substituting $\delta^{2}=k \epsilon$ into the equations, then from Eq.(2.29), Eq.(2.30) and Eq.(2.31), we have that

$$
\begin{gathered}
-u_{\tau}=\epsilon u\left(\epsilon u_{\xi}+g(\xi) u_{\tau}\right)+\epsilon v u_{r}+\epsilon p_{\xi}+g(\xi) p_{\xi}=0, \\
k \epsilon\left[\epsilon-v_{\tau}+\epsilon u\left(\epsilon v_{\xi}+g(\xi) v_{\tau}\right) \epsilon v v_{r}\right]+p_{r}=0, \\
\epsilon u_{\xi}+g(\xi) u_{\tau}+v_{r}+\frac{1}{r} v=0 .
\end{gathered}
$$

And from Eq.(2.33)-Eq.(2.34), we have

$$
\begin{gathered}
v=-\eta_{\tau}+\epsilon r_{0 \xi} u+\epsilon\left(\epsilon \eta_{\xi}+g(\xi) \eta_{\tau}\right) u, \\
\alpha k \epsilon \eta_{\tau \tau}=p^{w}-2 \frac{E(\xi)}{r_{0}^{2}} \eta,
\end{gathered}
$$

when $r=r^{w}$. To solve the equations for $\eta$, we express the $\eta, u, v$, and $p$ in the following form

$$
\begin{gathered}
\eta=\eta_{0}+\epsilon \eta_{1}+O\left(\epsilon^{2}\right), \quad v=v_{0}+\epsilon v_{1}+O\left(\epsilon^{2}\right), \\
u=u_{0}+\epsilon u_{1}+O\left(\epsilon^{2},\right), \quad p=p_{0}+\epsilon p_{1}+O\left(\epsilon^{2}\right) .
\end{gathered}
$$


We collect the terms of zero-order from Eq.(2.29), Eq.(2.30), Eq.(2.31),Eq.(2.33) and Eq.(2.34), and we obtain:

$$
\begin{gathered}
-u_{0 \tau}+g(\xi) p_{0 \tau}=0, \\
p_{0 r}=0, \\
g(\xi) u_{0 \tau}+v_{0 r}+\frac{1}{r} v_{0}=0,
\end{gathered}
$$

when $0 \leq r \leq r^{w}$. We also have that for $r=r^{w}=r_{0}+\epsilon \eta$

$$
\begin{gathered}
v_{0}=-\eta_{\tau}, \\
0=p_{0}-2 \frac{E(\xi)}{r_{0}^{2}} \eta_{0} .
\end{gathered}
$$

From Eq.(2.44), we observe that

$$
p_{0}=p_{0}\left(r^{w}\right)=2 \frac{E(\xi)}{r_{0}^{2}} \eta_{0}
$$

Substituting Eq.(2.48) into Eq.(2.43), one obtains

$$
u_{0 \tau}=2 g(\xi) \frac{E(\xi)}{r_{0}^{2}} \eta_{0 \tau}
$$

Combining Eq.(2.49) and Eq.(2.45), we have

$$
v_{0}=-r g(\xi) \frac{E(\xi)}{r_{0}^{2}} \eta_{0 \tau}
$$

Comparing the Eq.(2.50) with Eq.(2.46), we have that

$$
g(\xi)=\frac{r_{0}^{1 / 2}}{E(\xi)^{1 / 2}} .
$$

Thus the $g$ has been determined here. Additionally, we also have the following relations

$$
v_{0}=-\frac{r}{r_{0}} \eta_{0 \tau}
$$


14CHAPTER 2. MODEL EQUATIONS FOR FLUID FLOW IN ELASTIC VESSELS

$$
\begin{gathered}
p_{0}=2 \frac{E(\xi)}{r_{0}^{2}} \eta_{0}, \\
u_{0 \tau}=2 \frac{E(\xi)^{1 / 2}}{r_{0}^{3 / 2}} \eta_{0 \tau} .
\end{gathered}
$$

We observe that $u_{0 \tau}$ is independent of $\mathrm{r}$.

By collecting the first-order terms of Eq.(2.29), Eq.(2.30), Eq.(2.31),Eq.(2.33) and Eq.(2.34), one obtains

$$
\begin{aligned}
-u_{1 \tau}+g(\tau) u_{0} u_{0 \tau}+v_{0} v_{0 r}+p_{0 \xi}+g(\xi) p_{1 \tau} & =0, \\
-k v_{0 \xi}+p_{1 r} & =0, \\
u_{0 \xi}+g(\xi) u_{1 \tau}+v_{1 r}+\frac{1}{r} v_{1} & =0 .
\end{aligned}
$$

And for $r=r^{w}$, we have

$$
\begin{gathered}
v_{1}=-\eta_{1 \tau}+r_{0 \xi} u_{0}+g \tau u_{0} \eta_{0 \tau}, \\
\alpha k \eta_{0 \tau \tau}=p_{1}^{w}-2 \frac{E(\xi)}{r_{0}^{2}} \eta_{1} .
\end{gathered}
$$

By comparing Eq.(2.52) and Eq.(2.56), we have

$$
p_{1 r}=-k \frac{r}{r_{0}} \eta_{0 \tau \tau}
$$

We integrate both sides of Eq.(2.60), and obtain:

$$
p_{1}(r)=-k \frac{r^{2}}{2 r_{0}} \eta_{0 \tau \tau}+p_{1}(0) .
$$

If we take $r=r_{0}$, we will have

$$
p_{1}^{w}(\xi, \tau)=-k \frac{r_{0}}{2} \eta_{0 \tau \tau}+p_{1}(\xi, \tau, 0) .
$$

Combining Eq.(2.62) and Eq.(2.59), one obtains

$$
-\eta_{1}=\frac{r_{0}^{2}}{2 E(\xi)}\left[\alpha+\frac{r_{0}}{2}\right] k \eta_{0 \tau \tau}-\left.\frac{r_{0}^{2}}{2 E(\xi)} p_{1}\right|_{r=0} .
$$


Then we combine Eq.(2.60) and Eq.(2.58) to:

$$
v_{0}^{w}=\frac{r_{0}^{2}}{2 E(\xi)}\left[\alpha+\frac{r_{0}}{2}\right] k \eta_{0 \tau \tau \tau}-\left.\frac{r_{0}^{2}}{2 E(\xi)} p_{1 \tau}\right|_{r=0}+r_{0 \xi} u_{0}^{w}+\frac{r_{0}^{1 / 2}}{E(\xi)^{1 / 2}} u_{0}^{w} \eta_{0 \tau} .
$$

By combining Eq.(2.55) and Eq.(2.56), we can eliminate $u_{1 \tau}$ as follows:

$$
\begin{aligned}
\frac{1}{r}\left(r v_{1}\right)_{r}=-u_{0 \xi} & -\frac{r_{0}}{E(\xi)} u_{0} u_{0 \tau}+\frac{r}{r_{0}^{1 / 2} E(\xi)^{1 / 2}} u_{0} r \eta_{0 \tau} \\
& -2 \frac{r_{0}^{1 / 2}}{E(\xi)^{1 / 2}}\left[\frac{E(\xi)}{r_{0}^{2}} \eta_{0}\right]_{\tau}-\frac{r_{0}}{E(\xi)} p_{1 \tau} .
\end{aligned}
$$

For convenience, we take

$$
Q_{0}=\int_{0}^{r_{0}(\xi)} r u_{0} d r
$$

Then for $r=r_{0}$, we can integrate Eq.(2.65) and apply the Leibniz rule. We obtain that

$$
\begin{aligned}
v_{1}^{w}= & \frac{1}{r_{0}} Q_{0 \xi}+r_{0 \xi} u_{0}^{w}-\frac{2}{r_{0}^{3 / 2} E(\xi)^{1 / 2}} Q_{0} \eta_{o \tau}+\frac{r_{0}^{1 / 2}}{E(\xi)^{1 / 2}} u_{0}^{w} \eta_{0 \tau} \\
& -\frac{r_{0}^{3 / 2}}{E(\xi)^{1 / 2}}\left[\frac{E(\xi)}{r_{0}^{2}} \eta_{0}\right]_{\tau}+k \frac{r_{0}^{3}}{8 E(\xi)} \eta_{0 \tau \tau \tau}-\left.\frac{r_{0}^{2}}{2 E(\xi)} p_{1 \tau}\right|_{r=0} .
\end{aligned}
$$

Comparing the equation above with Eq.(2.64), we have

$$
\begin{array}{r}
\frac{E(\xi)^{1 / 2}}{r_{0}^{1 / 2}} \eta_{0 \xi}+\frac{r_{0}^{3 / 2}}{E(\xi)^{1 / 2}}\left[\frac{E(\xi)}{r_{0}^{2}}\right]_{\xi} \eta_{0}+k \frac{r_{0}^{2}}{E(\xi)}\left[\alpha+\frac{r_{0}}{4} \eta_{0 \tau \tau \tau}\right. \\
\frac{1}{r_{0}} Q_{0 \xi}+\frac{2}{r_{0}^{3 / 2} E(\xi)^{1 / 2}} Q_{0} \eta_{0 \tau}=0
\end{array}
$$

By Eq.(2.54), we have

$$
Q_{o \tau}=E(\xi)^{1 / 2} r_{0}^{1 / 2} \eta_{0 \tau}
$$

Thus, $Q_{0}$ can be expressed as a linear function of $\eta_{0}$

$$
Q_{0}(\xi, \tau)=E(\xi)^{1 / 2} r_{0}^{1 / 2} \eta_{0}(\xi, \tau)+f(\xi) .
$$


Then, we can determine $f$ by the initial condition for the blood vessel by setting $\mathrm{t}=0$. Then we have the equation

$$
\begin{array}{r}
\eta_{o \xi}+\frac{3}{4}\left(\frac{E_{\xi}(\xi)}{E(\xi)}-\frac{r_{0 \xi}(\xi)}{r_{0}(\xi)}\right) \eta_{0}+-\frac{3}{2} \frac{1}{r_{0}^{1 / 2} E(\xi)^{1 / 2}} \eta_{0} \eta_{0 \tau} \\
+\frac{k}{2} \frac{r_{0}^{5 / 2}}{E(\xi)^{3 / 2}}\left(\alpha+\frac{r_{0}}{4}\right) \eta_{0 \tau \tau \tau}+\frac{1}{r_{0}^{1 / 2} E(\xi)^{1 / 2}} f^{\prime}(\xi)+\frac{2}{r_{0} E(\xi)} f(\xi) \eta_{0 \tau}=0 .
\end{array}
$$

Consider the initial condition of quiescent state, which implied that $f(\xi)=$ 0 . If $E(\xi) \equiv 0$, then we can simplify the equation to

$$
\eta_{0 \xi}-\frac{3}{4} \eta_{0}+\frac{3}{2} r_{0}^{-1 / 2} \eta_{0} \eta_{0 \tau}+\frac{k}{2} r_{0}^{5 / 2}\left(\alpha+\frac{r_{0}}{4}\right) \eta_{0 \tau \tau \tau}=0 .
$$

If the radius of the unstressed vessel is constant, we have

$$
\eta_{0 \xi}+\frac{3}{2} r_{0}^{-1 / 2} \eta_{0} \eta_{0 \tau}+\frac{k}{2} r_{0}^{5 / 2}\left(\alpha+\frac{r_{0}}{4}\right) \eta_{0 \tau \tau \tau}=0
$$

\subsection{A 1-D Boussinesq system}

In this section we review the derivation of a bidirectional Boussinesq system for fluid flow in elastic vessel, [6]. From the conservation of mass and momentum, we obtain:

$$
\begin{gathered}
\eta_{t}+\eta_{x} u+\frac{1}{2}\left(\eta+r_{0}\right) u_{x}=0, \\
u_{t}+u u_{x}+\frac{1}{\rho} p_{x}=0 .
\end{gathered}
$$

And we also have Eq.(2.26). Then we obtain by substituting Eq.(2.27) into Eq.(2.74):

$$
\begin{gathered}
\eta_{t}+\eta_{x} u+\frac{1}{2}\left(\eta+r_{0}\right) u_{x}=0, \\
u_{t}+u u_{x}+\frac{E h}{\rho r_{0}^{2}} \eta_{x}+\frac{\rho_{w} h}{\rho} \eta_{x t t}=0 .
\end{gathered}
$$

Next we take the following non-dimensional quantities: 


$$
\begin{array}{r}
\eta^{*}=\frac{\eta}{a}, \quad x^{*}=\frac{x}{\lambda}, \\
u^{*}=\frac{u}{C_{0}}, \quad t^{*}=\frac{t}{\tau}, \\
C_{0}=\frac{a}{r_{0}} \sqrt{\frac{2 E_{\sigma} h}{\rho r_{0}}}, \quad \tau=\frac{2 a \lambda}{r_{0} C_{0}} . \\
\epsilon=\frac{2 a}{r_{0}}, \quad \delta^{2}=\frac{\rho_{2} h r_{0}}{2 \lambda^{2} \rho} .
\end{array}
$$

By substituting Eq.(2.77) into Eq.(2.75) and Eq.(2.76), we obtain the new system with non-dimensional variables:

$$
\begin{aligned}
& \eta_{t}+\epsilon \eta_{x} u+\frac{1}{2} \epsilon \eta u_{x}+u_{x}=0, \\
& u_{t}+\eta_{x}+\epsilon u u_{x}-\delta^{2} u_{x x t}=0,
\end{aligned}
$$

This is a Boussinesq type system but as we shall see later is not asymptotic. One can derive further unidirectional models from these equations, such as the BBM and $\mathrm{KdV}$ equations. The details of this derivation is postponed for later, to be exposed in the correct setting. The KdV equation is:

$$
\eta_{t}+\frac{1}{2} \sqrt{\frac{2 E h}{\rho r_{0}}} \eta+\frac{5}{4 r_{0}} \sqrt{\frac{2 E h}{\rho r_{0}}} \eta \eta_{x}+\frac{\rho_{w} h r_{0}}{4 \rho} \sqrt{\frac{E h}{2 \rho r_{0}}} \eta_{x x x}=0 .
$$

while the BBM equation is:

$$
\eta_{t}+\frac{1}{2} \sqrt{\frac{2 E h}{\rho r_{0}}} \eta+\frac{5}{4 r_{0}} \sqrt{\frac{2 E h}{\rho r_{0}}} \eta \eta_{x}-\frac{\rho_{w} h r_{0}}{4 \rho} \eta_{x x t}=0
$$


18CHAPTER 2. MODEL EQUATIONS FOR FLUID FLOW IN ELASTIC VESSELS 


\section{Chapter 3}

\section{Asymptotic derivation of Boussinesq systems}

In this chapter we derive asymptotically Boussinesq systems for fluid flow in elastic vessels. Because these systems are weakly non-linear and weakly dispersive we expect then to have solitary waves as special solutions. We explore some possibilities by construction analytical solutions.

\subsection{Boussnesq model}

Now we go back to the Euler equations with scaled variables.

$$
\begin{array}{r}
u_{t}+\epsilon u u_{x}+\epsilon v u_{r}+p_{x}=0, \\
\delta^{2}\left[v_{t}+\epsilon u v_{x}+\epsilon v v_{r}\right]+p_{r}=0, \\
r u_{x}+(r v)_{r}=0, \\
v\left(r^{w}\right)=\eta_{t}+r_{x}^{w} u, \\
\alpha \delta^{2} \eta_{t t}=p^{w}-\beta(x) \eta .
\end{array}
$$

In this chapter we will denote $\bar{\beta}(x)$ and $\bar{\alpha}$ the dimensional quatities defined by relations:

$$
\beta(x)=\frac{2 R^{2} \rho}{h} \bar{\beta}(x), \quad \alpha=\bar{\alpha} / R
$$


In order to simplify the model and derive Bousinesq systems, we assume that the fluid flow is irrotational. After scaling, the irrotationality condition becomes [17]:

$$
\delta^{2} v_{x}=u_{r}
$$

Integrating both sides of Eq.(3.6) from $r$ to $r^{w}$, we have

$$
\int_{r}^{r^{w}} u_{r^{\prime}} d r^{\prime}=\delta^{2} \int_{r}^{r^{w}} v_{x} d r^{\prime}
$$

Then

$$
u(r)=u\left(r^{w}\right)-\delta^{2} \int_{r}^{r^{w}} v_{x} d r^{\prime}
$$

which also implies that

$$
u(r)=u\left(r^{w}\right)+O\left(\delta^{2}\right)
$$

and

$$
u_{r}(r)=O\left(\delta^{2}\right)
$$

By applying the Leibniz rule, we have

$$
\int_{r}^{r^{w}} v_{x} d r^{\prime}=\left(\int_{r}^{r^{w}} v d r^{\prime}\right)_{x}-r_{x}^{w} v\left(r^{w}\right) .
$$

Similarly, by the Lebniz rule, we have

$$
\frac{\partial}{\partial x} \int_{r}^{r^{w}} r^{\prime} u d r^{\prime}=r_{x}^{w} r^{w} u\left(r^{w}\right)+\int_{r}^{r^{w}} r^{\prime} u_{x} d r^{\prime},
$$

For convenience, we define that

$$
Q(x, r, t)=\frac{1}{r} \int_{0}^{r} s u(x, s, t) d s .
$$

Comparing the above relation with Eq.(3.7), we observe

$$
Q=\frac{r}{2} u\left(r^{w}\right)+O\left(\delta^{2}\right) .
$$


By Eq.(3.3), we obtain

$$
\begin{array}{r}
\frac{\partial}{\partial r}(r v)=-r u_{x}, \\
r v=-\int_{0}^{r} s u_{x} d s \\
v=-\frac{1}{r} \int_{0}^{r} s u_{x} d s .
\end{array}
$$

We then have:

$$
v=-Q_{x}(x, r, t) .
$$

Additionally, because of Eq.(3.13), we obtain

$$
v=-\frac{r}{2} u\left(r^{w}\right)+O\left(\delta^{2}\right)
$$

Then differentiating both sides of Eq.(3.16) with respect to $x$, and substituting the result into Eq.(3.7), one obtains

$$
\begin{aligned}
u(r) & =u\left(r^{w}\right)+\delta^{2} \int_{r}^{r^{w}} \frac{r}{2} u_{x x}\left(r^{w}\right)+O\left(\delta^{4}\right) \\
& =u\left(r^{w}\right)+\delta^{2} u_{x x} \frac{\left(r^{w}\right)^{2}-r^{2}}{4}+O\left(\delta^{4}\right) .
\end{aligned}
$$

By Eq.(3.16), we also have that

$$
v_{t}=-\frac{r}{2} u_{x t}+O\left(\delta^{2}\right)
$$

Combing Eq.(3.2) and the relation we derived above, we obtain

$$
p_{r}=\delta^{2} \frac{r}{2} u_{x t}\left(r^{w}\right)+O\left(\epsilon \delta^{2}, \delta^{4}\right),
$$

which leads to:

$$
\begin{aligned}
p\left(r^{w}\right)-p(r) & =\delta^{2} u_{x t}\left(r^{w}\right) \int_{r}^{r^{w}} \frac{s}{2} d s+O\left(\epsilon \delta^{2}, \delta^{4}\right), \\
& =\delta^{2} u_{x t}\left(r^{w}\right) \frac{\left(r^{w}\right)^{2}-r^{2}}{4}+O\left(\epsilon \delta^{2}, \delta^{4}\right) .
\end{aligned}
$$


Then we have the following equation

$$
p(r)=p\left(r^{w}\right)-\delta^{2} u_{x t}\left(r^{w}\right) \frac{\left(r^{w}\right)^{2}-r^{2}}{4}+O\left(\epsilon \delta^{2}, \delta^{4}\right)
$$

If we differentiate both sides of the equation above, we obtain that

$$
p_{x}(r)=p_{x}^{w}-\delta^{2} u_{x x t}\left(r^{w}\right) \frac{\left(r^{w}\right)^{2}-r^{2}}{4}-\delta^{2} u_{x t} \frac{r^{w} r_{x}^{w}}{2}+O\left(\epsilon \delta^{2}, \delta^{4}\right) .
$$

Combining Eq.(3.9) and Eq.(3.1), we have that

$$
u_{t}+\epsilon u u_{x}+p_{x}=O\left(\epsilon \delta^{2}\right)
$$

We substitute Eq.(3.17) and Eq.(3.22) into the relation above, to obtain

$$
\begin{array}{r}
u_{t}\left(r^{w}\right)+\delta^{2} u_{x x t} \frac{\left(r^{w}\right)^{2}-r^{2}}{4}+\epsilon u\left(r^{w}\right) u_{x}\left(r^{w}\right)+p_{x}\left(r^{w}\right) \\
-\delta^{2} u_{x x t} \frac{\left(r^{w}\right)^{2}-r^{2}}{4}-\delta^{2} u_{x t} \frac{r^{w} r_{x}^{w}}{2}=O\left(\epsilon \delta^{2}, \delta^{4}\right) .
\end{array}
$$

Combining Eq.(3.4) and Eq.(3.16), we have

$$
-\frac{r^{w}}{2} u_{x}\left(r^{w}\right)=\eta_{t}+r_{x}^{w} u+O\left(\delta^{2}\right)
$$

thus

$$
\eta_{t}=-\frac{r^{w}}{2} u_{x}-r_{x}^{w} u+O\left(\delta^{2}\right) .
$$

Substituting Eq.(3.5) and Eq.(3.25) into Eq.(3.24), we have

$$
\begin{array}{r}
u_{t}+\epsilon u u_{x}+(\beta(x) \eta)_{x}-\alpha \delta^{2}\left(\frac{r^{w}}{2} u_{x}\right)_{x t}-\alpha \delta^{2}\left(r_{x}^{w} u\right)_{x t} \\
-\delta^{2} u_{x t} \frac{r^{w} r_{x}^{w}}{2}=O\left(\epsilon \delta^{2}, \delta^{4}\right),
\end{array}
$$

where $\alpha=\frac{\rho_{w} h}{\rho R}$ and $\beta(x)=\frac{2 E(x)}{r_{0}^{2}(x)}$. We can substitute $r^{w}=r_{0}+\epsilon \eta$ into 
Eq.(3.26), then we obtain

$$
\begin{array}{r}
u_{t}+\epsilon u u_{x}+(\beta(x) \eta)_{x}-\alpha \delta^{2}\left(\frac{r_{0}}{2} u_{x}\right)_{x t}-\alpha \delta^{2}\left(r_{0 x} u\right)_{x t} \\
-\delta^{2} u_{x t} \frac{r_{0} r_{0 x}}{2}=O\left(\epsilon \delta^{2}, \delta^{4}\right),
\end{array}
$$

For Eq.(3.27), we can also discard the high-order terms and go back to the dimensional form, which is

$$
\begin{array}{r}
u_{t}+u u_{x}+\left(\frac{E_{\sigma}(x) h}{r_{0}^{2} \rho} \eta\right)_{x}-\frac{\rho^{w} h}{\rho}\left(\frac{r_{0}}{2} u_{x}\right)_{x t}-\frac{\rho^{w} h}{\rho}\left(r_{0 x} u\right)_{x t} \\
-u_{x t} \frac{r_{0} r_{0 x}}{2}=0
\end{array}
$$

where the tildes in the dimensional variables have been omitted for convenience.

Integrating both sides of Eq.(3.3), gives

$$
r^{w} v\left(r^{w}\right)=-\int_{0}^{r^{w}} s u_{x} d s .
$$

Then we substitute Eq.(3.4) into the left-hand side of the relation above, and we have

$$
r^{w}\left(\eta_{t}+r_{x}^{w} u\right)=-\int_{0}^{r^{w}} s u_{x} d s
$$

Then we substitute Eq.(3.17) into the Equation above, and we have

$$
\begin{aligned}
r^{w}\left(\eta_{t}+r_{x}^{w} u\right)= & -\int_{0}^{r^{w}} r\left[u_{x}\left(r^{w}\right)+\delta^{2}\left(u_{x x}\left(r^{w}\right) \frac{\left(r^{w}\right)^{2}-r^{2}}{4}\right)_{x}\right]+O\left(\delta^{4}\right) \\
= & -\int_{0}^{r^{w}} s u_{x}\left(r^{w}\right) d s-\delta^{2} \int_{0}^{r^{w}} s u_{x x x}\left(r^{w}\right) \frac{\left(r^{w}\right)^{2}-r^{2}}{4} \\
& -\delta^{2} \int_{0}^{r^{w}} s u_{x x}\left(r^{w}\right) \frac{r^{w} r_{x}^{w}}{2}+O\left(\text { delt }^{4}\right) \\
= & -\frac{\left(r^{w}\right)^{2}}{2} u_{x}+\delta^{2} u_{x x x} \frac{\left(r^{w}\right)^{3}}{16}-\delta^{2} u_{x x} \frac{\left(r^{w}\right)^{3} r_{x}^{w}}{4}+O\left(\delta^{4}\right)
\end{aligned}
$$


Thereby we obtain the second system equation

$$
\eta_{t}+r_{x}^{w} u+\frac{r^{w}}{2} u_{x}+\delta^{2} u_{x x} \frac{\left(r^{w}\right)^{2} r_{x}^{w}}{4}+\delta^{2} u_{x x x} \frac{\left(r^{w}\right)^{3}}{16}=O\left(\delta^{4}\right)
$$

we can then discard the high order terms and go back to the dimensional form. For convenience, the tilde above the dimensional variables are discarded

$$
\eta_{t}+r_{x}^{w} u+\frac{r^{w}}{2} u_{x}+\frac{r^{w 2} r_{x}^{w}}{4} u_{x x}+\frac{r^{w 3}}{16} u_{x x x}=0 .
$$

For Eq.(3.30), we make the substitution $r^{w}=r_{0}+\epsilon \eta$ and discard the highorder terms, then we have

$$
\eta_{t}+r_{0 x} u+\epsilon \eta_{x} u+\frac{r_{0}}{2} u_{x}+\frac{\epsilon \eta}{2} u_{x}+\delta^{2} u_{x x} \frac{\left(r_{0}\right)^{2} r_{0 x}}{4}+\delta^{2} u_{x x x} \frac{\left(r_{0}\right)^{3}}{16}=0 .
$$

The equation above can also be transformed to dimensional form

$$
\eta_{t}+r_{0 x} u+\eta_{x} u+\frac{r_{0}}{2} u_{x}+\frac{\eta}{2} u_{x}+u_{x x} \frac{\left(r_{0}\right)^{2} r_{0 x}}{4}+u_{x x x} \frac{\left(r_{0}\right)^{3}}{16}=0 .
$$

Now we obtain the system of variable radius system, which is

$$
\begin{aligned}
u_{t}+\epsilon u u_{x}+(\beta(x) \eta)_{x}- & \alpha \delta^{2}\left(\frac{r^{0}}{2} u_{x}\right)_{x t}-\alpha \delta^{2}\left(r_{0 x} u\right)_{x t} \\
- & \delta^{2} u_{x t} \frac{r_{0} r_{0 x}}{2}=O\left(\epsilon \delta^{2}, \delta^{4}\right),
\end{aligned}
$$

$\eta_{t}+r_{0 x} u+\epsilon \eta_{x} u+\frac{r_{0}}{2} u_{x}+\frac{\epsilon \eta}{2} u_{x}+\delta^{2} u_{x x} \frac{\left(r_{0}\right)^{2} r_{0 x}}{4}+\delta^{2} u_{x x x} \frac{\left(r_{0}\right)^{3}}{16}=O\left(\epsilon \delta^{2}, \delta^{4}\right)$.

The system above also have the dimensional form:

$$
\begin{array}{r}
u_{t}+u u_{x}+\left(\frac{E_{\sigma}(x) h}{r_{0}^{2} \rho} \eta_{x}-\frac{\rho^{w} h}{\rho}\left(\frac{r_{0}}{2} u_{x}\right)_{x t}-\frac{\rho^{w} h}{\rho}\left(r_{0 x} u\right)_{x t}\right. \\
-u_{x t} \frac{r_{0} r_{0 x}}{2}=0,
\end{array}
$$




$$
\eta_{t}+r_{0 x} u+\eta_{x} u+\frac{r_{0}}{2} u_{x}+\frac{\eta}{2} u_{x}+u_{x x} \frac{\left(r_{0}\right)^{2} r_{0 x}}{4}+u_{x x x} \frac{\left(r_{0}\right)^{3}}{16}=0 .
$$

\subsection{The equations at the center of the vessel}

For convenience to do the comparison with the result in Chapter 6, we rewrite Eq.(3.30) in the following form:

$$
\eta_{t}+r_{0 x} u^{w}+\epsilon \eta_{x} u^{w}+\frac{r_{0}}{2} u_{x}^{w}+\frac{\epsilon \eta}{2} u_{x}^{w}+\delta^{2} u_{x x}^{w} \frac{\left(r_{0}\right)^{2} r_{0 x}}{4}+\delta^{2} u_{x x x}^{w} \frac{\left(r_{0}\right)^{3}}{16}=0
$$

which is from last section of this chapter. The upper label $w$ is used to indicate put to notice that the velocity is essentially the horizontal velocity at the vessel wall. If we denote the horizontal velocity in the center of the vessel wall by $u_{0}$, and combine with Eq.(3.16), one obtains that:

$$
u^{w}=u_{0}-\delta^{2} u_{x x}^{w} \frac{\left(r^{w}\right)^{2}}{4}+O\left(\delta^{4}\right) .
$$

$u_{0}$ is the horizontal velocity ar the center of the vessel. Since we already have

$$
u^{w}=u(r)+O\left(\epsilon, \delta^{2}\right), \quad r^{w}=r_{0}+\epsilon \eta
$$

we can rewrite the above equation as

$$
u^{w}=u_{0}-\delta^{2} u_{0 x x} \frac{\left(r_{0}\right)^{2}}{4}+O\left(\epsilon \delta^{2}, \delta^{4}\right)
$$

Now we have

$$
\begin{gathered}
r_{0 x} u^{w}=r_{0 x} u_{0}-\frac{1}{4} \delta^{2} r_{0 x} r_{0}^{2} u_{0 x x}, \\
\frac{r_{0}}{2} u_{x}^{w}=\frac{r_{0}}{2} u_{0 x}-\frac{\delta^{2}}{8} r_{0}^{3} u_{0 x x x}-\frac{1}{4} \delta^{2} r_{0 x} r_{0}^{2} u_{0 x x} .
\end{gathered}
$$

Then for Eq.(3.38), we have 
$\eta_{t}+r_{0 x} u_{0}+\epsilon \eta_{x} u_{0}+\frac{r_{0}}{2} u_{0} x+\frac{\epsilon \eta}{2} u_{0} x-\delta^{2} u_{0 x x} \frac{\left(r_{0}\right)^{2} r_{0 x}}{4}-\delta^{2} u_{0 x x x} \frac{\left(r_{0}\right)^{3}}{16}=O\left(\epsilon \delta^{2}\right)$.

Now we study the equation of Eq.(3.23):

$$
u_{t}+\epsilon u u_{x}+p_{x}=O\left(\epsilon \delta^{2}\right)
$$

consider when $r=0$ :

$$
u_{0 t}+\epsilon u_{0 x} u_{0}+p_{0 x}=O\left(\epsilon \delta^{2}\right),
$$

where $p_{0 x}=\left.p_{x}\right|_{r=0}$. For the pressure, we consider Eq.(3.22):

$$
p_{x}(r)=p_{x}^{w}-\delta^{2} u_{x x t}\left(r^{w}\right) \frac{\left(r^{w}\right)^{2}-r^{2}}{4}-\delta^{2} u_{x t} \frac{r^{w} r_{x}^{w}}{2}+O\left(\epsilon \delta^{2}, \delta^{4}\right) .
$$

Taking $r=0$ for Eq.(3.43), we have:

$$
p_{x}(r=0)=p_{x}\left(r^{w}\right)-\delta^{2} u_{x x t}\left(r^{w}\right) \frac{\left(r^{w}\right)^{2}}{4}-\delta^{2} u_{x t} \frac{r^{w} r_{x}^{w}}{2}+O\left(\epsilon \delta^{2}, \delta^{4}\right) .
$$

Since we have By Eq.(3.8), we know that:

$$
\begin{gathered}
u_{x x t}\left(r^{w}\right)=u_{0 x x t}+O\left(\delta^{2}\right), \\
u_{x t}\left(r^{w}\right)=u_{0 x t}+O\left(\delta^{2}\right),
\end{gathered}
$$

where $u_{0 x x t}$ denotes $\left.\left.u_{x x t}\right|_{r=0}\right)$ and $u_{0 x t}$ denotes $\left.\left.u_{x t}\right|_{r=0}\right)$. Then for Eq.(3.44), we have

$$
p_{x}(r=0)=p_{x}^{w}-\delta^{2} u_{0 x x t} \frac{\left(r^{w}\right)^{2}}{4}-\delta^{2} u_{0 x t} \frac{r^{w} r_{x}^{w}}{2}+O\left(\epsilon \delta^{2}, \delta^{4}\right) .
$$

Since $r^{w}=r_{0}+\epsilon \eta$, for Eq.(3.45) we have

$$
p_{x}(r=0)=p_{x}^{w}-\delta^{2} u_{0 x x t} \frac{\left(r_{0}\right)^{2}}{4}-\delta^{2} u_{0 x t} \frac{r_{0} r_{0 x}}{2}+O\left(\epsilon \delta^{2}, \delta^{4}\right) .
$$

Now we have the following equation after combing Eq.(3.42) and Eq.(3.46) 
after discarding the high-order terms:

$$
u_{0 t}+\epsilon u_{0} u_{0 x}+p_{x}^{w}-\delta^{2} u_{0 x x t} \frac{\left(r_{0}\right)^{2}}{4}-\delta^{2} u_{0 x t} \frac{r_{0} r_{0 x}}{2}=0 .
$$

Eq.(3.47) is very useful to make comparison with the models.

\subsection{Solitary waves of the Boussnesq system}

Solitary wave solutions can propagate without change in shape and speed in a uniform medium. For this reason we consider the case where $r_{0}$ and $E(x)$ are constant. Then Eq.(3.34) and Eq.(3.35) become

$$
\begin{gathered}
\eta_{t}+\epsilon \eta_{x} u+\frac{r_{0}}{2} u_{x}+\frac{\epsilon}{2} \eta u_{x}+\delta^{2} \frac{r_{0}^{3}}{16} u_{x x x}=O\left(\epsilon^{2}, \delta^{4}\right), \\
u_{t}+\epsilon u u_{x}+(\beta \eta)_{x}-\alpha \frac{\delta^{2}}{2} r_{0} u_{x x t}=O\left(\epsilon^{2}, \delta^{4}\right) .
\end{gathered}
$$

In order to find the solitary wave solution for Eq.(3.48) and Eq.(3.48), we take

$$
\begin{aligned}
& \eta=\eta(x-c t), \\
& u=u(x-c t),
\end{aligned}
$$

where $\mathrm{c}$ is a constant [7]. Let $y=x-c t$, and consider the derivatives with respect to $y$ of $\eta$ and u. Thereby for Eq.(3.48) and Eq.(3.49) we obtain

$$
\begin{gathered}
-c \eta^{\prime}+\epsilon \eta^{\prime} u+\frac{r_{0}}{2} u^{\prime}+\frac{\epsilon}{2} \eta u^{\prime}+\delta^{2} \frac{r_{0}^{3}}{16} u^{\prime \prime \prime}=0, \\
-c u^{\prime}+\epsilon u u^{\prime}+\beta \eta^{\prime}+c \alpha \frac{\delta^{2}}{2} r_{0} u^{\prime \prime \prime}=0 .
\end{gathered}
$$

To solve the previous equations, we assume that $u=b \eta$, thus

$$
\begin{gathered}
-c \eta^{\prime}+\epsilon \eta^{\prime} b \eta+\frac{r_{0}}{2} b \eta^{\prime}+\frac{\epsilon}{2} \eta b \eta^{\prime}+\delta^{2} \frac{r_{0}^{3}}{16} b \eta^{\prime \prime \prime}=0, \\
-c b \eta^{\prime}+\epsilon b^{2} \eta \eta^{\prime}+\beta \eta^{\prime}+c \alpha \frac{\delta^{2}}{2} r_{0} b \eta^{\prime \prime \prime}=0 .
\end{gathered}
$$


Since the solution for the equations are solitary wave, as $y \rightarrow \infty, \eta, u \rightarrow 0$. Then we can integrate the equations above and rearrange the terms,

$$
\begin{aligned}
\left(\frac{r_{0} b}{2}-c\right) \eta+\frac{3 \epsilon b}{4} \eta^{2}+\frac{\delta^{2} r_{0}^{3}}{16} b \eta^{\prime \prime} & =0 \\
(\beta-c b) \eta+\frac{\epsilon b^{2}}{2} \eta^{2}+\frac{c \alpha \delta^{2} r_{0} b}{2} \eta^{\prime \prime} & =0
\end{aligned}
$$

Now we consider the two equations above are the same, or we will have trivial solution. So we must have that

$$
\begin{aligned}
\left(\frac{r_{0} b}{2}-c\right) \frac{\epsilon b^{2}}{2} & =\frac{3 \epsilon b}{4}(\beta-c b) \\
\frac{\delta^{2} r_{0}^{3}}{16} b \frac{\epsilon b^{2}}{2} & =\frac{3 \epsilon b}{4} \frac{c \alpha \delta^{2} r_{0} b}{2}
\end{aligned}
$$

Solving for the Eq.(3.51), we have that

$$
b=6 \sqrt{\frac{\alpha \beta}{r_{0}^{2}+12 r_{0} \alpha}}, \quad c=\frac{r_{0}^{2}}{2} \sqrt{\frac{\beta}{\alpha r_{0}^{2}+12 r_{0} \alpha^{2}}},
$$

Then we have solitary wave solution

$$
\begin{gathered}
\eta(x, t)=\frac{2 \sqrt{\beta}\left(6 \alpha-r_{0}\right) \sqrt{r_{0}^{2}+12 r_{0} \alpha}}{\epsilon \sqrt{\alpha}} \operatorname{sech}^{2}\left(X_{1}\right), \\
X_{1}=\sqrt{\frac{32\left(6 \alpha-r_{0}\right.}{3 \delta^{2} r_{0}^{3}}}\left(x+x_{0}-\frac{r_{0}^{2}}{2} \sqrt{\frac{\beta}{\alpha r_{0}^{2}+12 r_{0} \alpha^{2}}} t\right) .
\end{gathered}
$$

where $r_{0}$ is constant. 


\section{Chapter 4}

\section{A general system}

In this chapter we continue the derivation of the previous chapter. We derive a general system where the horizontal velocity is evaluated at any distance from the center of the tube. Special emphasis is paid on the case of constant radius vessels. We also derive unidirectional models and obtain the dissipative Boussinesq system by taking the viscosity effects into account.

\subsection{Further developments}

In the previous chapter, the velocity $u$ is evaluated on the wall, and is denotedby $u^{w}$. In order to derive the general system, we consider that

$$
u^{w}(x, t)=u(x, r, t)-\delta^{2} u_{x x}^{w}(x, r, t) \frac{\left(r^{w}\right)^{2}-r^{2}}{4}+O\left(\delta^{4}\right),
$$

which is from Eq.(3.17). Here we can take

$$
r=\theta r^{w}
$$

with $0 \leq \theta \leq 1$. Then we have the velocity for any $r$. Additionally, we denote $u\left(x, \theta r^{2}, t\right)$ by $u^{\theta}(x, t)$ for convenience. Then we have that

$$
u^{w}=u^{\theta}-\delta^{2} u_{x x}^{\theta} \frac{\left(1-\theta^{2}\right)\left(r^{w}\right)^{2}}{4}+O\left(\delta^{4}\right) .
$$


Following the derivation of the previous chapter, we have

$$
\begin{array}{r}
\eta_{t}+\frac{1}{2}\left(r_{0}+\epsilon \eta\right) u_{x}^{\theta}+\left(r_{0}+\epsilon \eta_{x}\right) u^{\theta}-\delta^{2} \frac{r_{0}^{2} r_{0 x}\left(1-2 \theta^{2}\right)}{4} u_{x x}^{\theta} \\
+\delta^{2} \frac{r_{0}^{3}\left(2 \theta^{2}-1\right)}{16} u_{x x x}^{\theta}=O\left(\epsilon \delta^{2}, \delta^{4}\right), \\
\left(1-\alpha \delta^{2} r_{0 x x}\right) u_{t}^{\theta}+[\beta(x) \eta]_{x}+\epsilon u^{\theta} u_{x}^{\theta}-\delta^{2} r_{0 x} \frac{3 \alpha+r_{0}}{2} u_{x t}^{\theta} \\
-\delta^{2} \frac{\left[3 \alpha-\left(1-\theta^{2}\right) r_{0}\right] r_{0}}{6} u_{x x t}^{\theta}=O\left(\epsilon \delta^{2}, \delta^{4}\right) .
\end{array}
$$

By Eq.(4.2) and Eq.(4.3), we also have the relations between the low-order terms:

$$
\begin{gathered}
\eta_{t}=\frac{1}{2} r_{0} u_{x}^{\theta}+r_{0 x} u^{\theta}+O\left(\epsilon, \delta^{2}\right), \\
u_{t}^{\theta}=-[\beta(x) \eta]_{x}+O\left(\epsilon, \delta^{2}\right) .
\end{gathered}
$$

From Eq.(4.4) we know that

$$
r_{0} u_{x x x}^{\theta}=-2 \eta_{x x t}-5 r_{0 x x} u_{x}^{\theta}-4 r_{0 x} u \theta_{x x}-2 r_{0 x x x} u^{\theta}+O\left(\epsilon, \delta^{2}\right),
$$

and by Eq.(4.5), we have

$$
u_{x x t}^{\theta}=-[\beta(x) \eta]_{x x x}+O\left(\epsilon, \delta^{2}\right)
$$

To derive the general system, we make the following substitution:

$$
\begin{aligned}
& u_{x x x}^{\theta}=\nu u_{x x x}^{\theta}+(1-\nu) u_{x x x}^{\theta}, \\
& u_{x x t}^{\theta}=\mu u_{x x t}^{\theta}+(1-\mu) u_{x x t}^{\theta},
\end{aligned}
$$

where $\mu, \nu, \in \mathbb{R}$. By Eq.(4.4), Eq.(4.5) and the above relations, we have the general system

$$
\begin{aligned}
\eta_{t}+ & \frac{1}{2}\left(r_{0}+\epsilon \eta\right) u_{x}^{\theta}+\left(r_{0 x}+\epsilon \eta_{x}\right) u^{\theta}-\delta^{2} A(x) u_{x x}^{\theta}+\delta^{2} B(x) u_{x x x}^{\theta}- \\
& \delta^{2} C(x)\left(5 r_{0 x x} u_{x}^{\theta}+4 r_{0 x} u_{x x}^{\theta}+2 r_{0 x x x} u^{\theta}+2 \eta_{x x t}\right)=O\left(\epsilon, \delta^{2}\right),
\end{aligned}
$$




$$
\begin{aligned}
(1- & \left.\alpha \delta^{2} r_{0 x x t}\right) u_{t}^{\theta}+[\beta(x) \eta]_{x}+\epsilon u^{\theta} u_{x}^{\theta}-\delta^{2} D(x) u_{x t}^{\theta} \\
& +\delta^{2} G(x)[\beta(x) \eta]_{x x x}-\delta^{2} F(x) u_{x x t}^{\theta}=O\left(\epsilon, \delta^{2}\right),
\end{aligned}
$$

where

$$
\begin{gathered}
A(x)=\frac{r_{0}^{2} r_{0 x}\left(1-2 \theta^{2}\right)}{4}, \quad B(x)=\frac{r_{0}^{3}\left(2 \theta^{2}-1\right) \nu}{16}, \\
C(x)=\frac{r_{0}^{2}\left(2 \theta^{2}-1\right)(1-\nu)}{16}, \quad D(x)=\frac{r_{0 x}\left(3 \alpha+r_{0}\right)}{2}, \\
G(x)=\frac{\left[2 \alpha+\left(1-\theta^{2}\right) r_{0}\right] r_{0} \mu}{4}, \quad F(x)=\frac{\left[2 \alpha+\left(1-\theta^{2}\right) r_{0}\right] r_{0}(1-\mu)}{4} .
\end{gathered}
$$

The dimensional form of Eq.(4.8) and Eq.(4.9) is:

$$
\begin{gathered}
\eta_{t}+\frac{1}{2}\left(r_{0}+\eta\right) u_{x}^{\theta}+\left(r_{0 x}+\eta_{x}\right) u^{\theta}-\bar{A}(x) u_{x x}^{\theta}+\bar{B}(x) u_{x x x}^{\theta}- \\
\bar{C}(x)\left(5 r_{0 x x} u_{x}^{\theta}+4 r_{0 x} u_{x x}^{\theta}+2 r_{0 x x x} u^{\theta}+2 \eta_{x x t}\right)=0, \\
\left(1-\bar{\alpha} r_{0 x x t}\right) u_{t}^{\theta}+[\bar{\beta}(x) \eta]_{x}+u^{\theta} u_{x}^{\theta}-\bar{D}(x) u_{x t}^{\theta} \\
+\bar{G}(x)[\bar{\beta}(x) \eta]_{x x x}-\delta^{2} \bar{F}(x) u_{x x t}^{\theta}=O\left(\epsilon, \delta^{2}\right),
\end{gathered}
$$

where

$$
\begin{gathered}
\bar{A}(x)=\frac{r_{0}^{2} r_{0 x}\left(3-2 \theta^{2}\right)}{4}, \quad \bar{B}(x)=\frac{r_{0}^{3}\left(2 \theta^{2}-1\right) \nu}{16}, \\
\bar{C}(x)=\frac{r_{0}^{2}\left(2 \theta^{2}-1\right)(1-\nu)}{16}, \\
\bar{D}(x)=\frac{r_{0 x}\left(3 \bar{\alpha}+r_{0}\right)}{2} \\
\bar{G}(x)=\frac{\left[2 \bar{\alpha}+\left(1-\theta^{2}\right) r_{0}\right] r_{0} \mu}{4}, \\
\bar{F}(x)=\frac{\left[2 \bar{\alpha}+\left(1-\theta^{2}\right) r_{0}\right] r_{0}(1-\mu)}{4} .
\end{gathered}
$$

Observe that if we take $\theta^{2}=\frac{1}{2}, \quad \mu=\nu=0$ for Eq.(4.8) and Eq.(4.9), we will obtain the simplest system:

$$
\eta_{t}+\frac{1}{2}\left(r_{0}+\epsilon \eta\right) u_{x}^{\theta}+\left(r_{0 x}+\epsilon \eta_{x}\right) u^{\theta}=O\left(\epsilon \delta^{2}, \delta^{4}\right)
$$




$$
\begin{array}{r}
\left(1-\alpha \delta^{2} r_{0 x x t}\right) u_{t}^{\theta}+[\beta(x) \eta]_{x}+\epsilon u^{\theta} u_{x}^{\theta}-\delta^{2} \frac{\left(3 \alpha+r_{0}\right) r_{0 x}}{2} u_{x t}^{\theta} \\
-\delta^{2} \frac{\left(4 \alpha+r_{0}\right) r_{0}}{8} u_{x x t}^{\theta}=O\left(\epsilon \delta^{2}, \delta^{4}\right) .
\end{array}
$$

We can also go back to the dimensional form for Eq.(4.12) and Eq.(4.13):

$$
\begin{gathered}
\eta_{t}+\frac{1}{2}\left(r_{0}+\eta\right) u_{x}^{\theta}+\left(r_{0 x}+\eta_{x}\right) u^{\theta}=0, \\
\left(1-\bar{\alpha} r_{0 x x t}\right) u_{t}^{\theta}+[\beta \overline{(x)} \eta]_{x}+u^{\theta} u_{x}^{\theta}-\frac{\left(3 \alpha+r_{0}\right) r_{0 x}}{2} u_{x t}^{\theta} \\
-\frac{\left(4 \bar{\alpha}+r_{0}\right) r_{0}}{8} u_{x x t}^{\theta}=0 .
\end{gathered}
$$

where

$$
\bar{\alpha}=\frac{\rho^{w} h}{\rho}, \quad \bar{\beta}=\frac{E(x) h}{r_{0}^{2} \rho} .
$$

Notice that the $E(x)$ here is the dimensional variable before scaling.

\subsection{The system with constant radius}

In this section we study the model with constant radius and Young's module. Then Eq.(4.8) and Eq.(4.9), we take the following form:

$$
\begin{array}{r}
\eta_{t}+\frac{1}{2}\left(r_{0}+\epsilon \eta\right) u_{x}^{\theta}+\epsilon \eta_{x} u^{\theta}+\delta^{2} r_{0}^{3} a u_{x x x}^{\theta}-\delta^{2} r_{0}^{2} b \eta_{x x t}=0 \\
u_{t}^{\theta}+\beta \eta_{x}+\epsilon u^{\theta} u_{x}^{\theta}+\delta^{2} r_{0}^{2} \beta c \eta_{x x x}-\delta^{2} r_{0}^{2} d u_{x x t}^{\theta}=0
\end{array}
$$

where

$$
\begin{gathered}
a=\frac{\left(2 \theta^{2}-1\right) \nu}{16}, \quad b=\frac{\left(2 \theta^{2}-1\right)(1-\nu)}{8}, \\
c=\frac{\left(2 \alpha+\left(1-\theta^{2}\right)\right) \mu}{4}, \quad d=\frac{\left(2 \alpha+\left(1-\theta^{2}\right)\right)(1-\mu)}{4} .
\end{gathered}
$$

In the previous parts, we saw that the form of $\alpha$ is

$$
\alpha=\frac{\rho^{w} h}{\rho R},
$$


where $R$ is the characteristic radius used in scaling. Now we can make some simplification since now the radius $r_{0}$ is a constant. Then we can take

$$
R=\frac{r_{0}}{2},
$$

therefore the system of Eq.(4.16) and Eq.(4.17) can be written in the following form:

$$
\begin{gathered}
\eta_{t}+u^{\theta}+\frac{1}{2} \epsilon \eta u_{x}^{\theta}+\epsilon \eta_{x} u^{\theta}+8 \delta^{2} a u_{x x x}^{\theta}-4 \delta^{2} b \eta_{x x t}=0 \\
u_{t}^{\theta}+\beta \eta_{x}+\epsilon u^{\theta} u_{x}^{\theta}+4 \delta^{2} \beta c \eta_{x x x}-4 \delta^{2} d u_{x x t}^{\theta}=0
\end{gathered}
$$

and the $\alpha$ is now of the form:

$$
\alpha=\frac{2 \rho^{w} h}{\rho r_{0}},
$$

which is a constant that has no relation with scaling variables. Since that the Young Module $E$ is scaled in for the non-dimensional form of $\beta$, we can take $\beta=1$ for convenience. In fact, we will have the form of:

$$
\begin{aligned}
& \eta_{x}+u_{t}=O\left(\epsilon, \delta^{2}\right), \\
& u_{x}+\eta_{x}=O\left(\epsilon, \delta^{2}\right),
\end{aligned}
$$

which occurs very often in the scaled Boussinesq system. Of course we also can take $R=r_{0}$. Then the system will be:

$$
\begin{gathered}
\eta_{t}+\frac{1}{2} u^{\theta}+\frac{1}{2} \epsilon \eta u_{x}^{\theta}+\epsilon \eta_{x} u^{\theta}+\delta^{2} a u_{x x x}^{\theta}-\delta^{2} b \eta_{x x t}=0, \\
u_{t}^{\theta}+\beta \eta_{x}+\epsilon u^{\theta} u_{x}^{\theta}+\delta^{2} \beta c \eta_{x x x}-\delta^{2} d u_{x x t}^{\theta}=0
\end{gathered}
$$

The dimensional form of the previous system with constant radius is:

$$
\begin{gathered}
\eta_{t}+\frac{1}{2} u_{x}^{\theta}+\eta_{x} u^{\theta}+r_{0}^{3} a u_{x x x}^{\theta}-r_{0}^{2} b \eta_{x x t}=0, \\
u_{t}^{\theta}+\frac{E h}{\rho r_{0}^{2}} \eta_{x}+u^{\theta} u_{x}^{\theta}+\frac{E h}{\rho r_{0}^{2}} r_{0}^{2} c \eta_{x x x}-r_{0}^{2} d u_{x x t}^{\theta}=0 .
\end{gathered}
$$


The linearisation of the system around the trivial solution is:

$$
\begin{gathered}
\eta_{t}+\frac{1}{2} r_{0} u_{x}^{\theta}+r_{0}^{3} a u_{x x x}^{\theta}-r_{0}^{2} b \eta_{x x t}=0, \\
u_{t}^{\theta}+\frac{E h}{\rho r_{0}^{2}} \eta_{x}+\frac{E h}{\rho r_{0}^{2}} r_{0}^{2} c \eta_{x x x}-r_{0}^{2} d u_{x x t}^{\theta}=0 .
\end{gathered}
$$

The linearized system will be used in the following chapters.

\subsection{One way propagation models}

In order to derive one-way propagation models, we consider the system of Eq.(4.14) and Eq.(4.15)

$$
\begin{gathered}
\eta_{t}+\frac{1}{2}\left(r_{0}+\eta\right) u_{x}^{\theta}+\left(r_{0 x}+\eta_{x}\right) u^{\theta}=0, \\
\left(1-\bar{\alpha} r_{0 x x t}\right) u_{t}^{\theta}+[\beta \overline{(x)} \eta]_{x}+u^{\theta} u_{x}^{\theta}-\frac{\left(3 \alpha+r_{0}\right) r_{0 x}}{2} u_{x t}^{\theta} \\
-\frac{\left(4 \bar{\alpha}+r_{0}\right) r_{0}}{8} u_{x x t}^{\theta}=0 .
\end{gathered}
$$

Now we take the Young modulus $E$ and the radius $r_{0}$ to be constant. In order to carry out the calculation, we use the scaling which was used in Eq.(2.77) in Chapter 2. Let $a$ be the characteristic displacement and $\lambda$ be the length of the vessel. Then we take the non-dimensional quantities

$$
\begin{array}{r}
\eta^{*}=\frac{\eta}{a}, \quad x^{*}=\frac{x}{\lambda}, \quad u^{*}=\frac{u}{C_{0}}, \\
t^{*}=\frac{t}{\tau}, \quad C_{0}=\frac{a}{r_{0}} \sqrt{\frac{2 E_{\sigma} h}{\rho r_{0}}}, \quad \tau=\frac{2 a \lambda}{r_{0} C_{0}} .
\end{array}
$$

Thus for the derivatives of the variables, we have

$$
\eta_{t}=\frac{\partial}{\partial t} \eta(x, t)=\frac{\partial}{\partial t} a \eta^{*}\left(x^{*}, t^{*}\right)=\frac{\partial \eta^{*}}{\partial t^{*}} \frac{\partial t^{*}}{\partial t}=\frac{a}{\tau} \eta_{t^{*}}^{*},
$$




$$
\begin{gathered}
\eta_{x}=\frac{a}{\lambda} \eta_{x^{*}}^{*}, \quad u_{x}=\frac{C_{0}}{\lambda} u_{x^{*}}^{*} \\
u_{t}=\frac{C_{0}}{\tau} u_{t^{*}}^{*}, \quad \eta_{x t t}=\frac{a}{\lambda} \frac{1}{\tau^{2}} \eta_{x^{*} x^{*} t^{*}}^{*}
\end{gathered}
$$

Set $\epsilon=\frac{a}{r_{0}}, \delta^{2}=\frac{r_{0}^{2}}{\lambda^{2}}$, where $\epsilon$ characterises the nonlinearity and $\delta^{2}$ the dispersion. We also assume Stokes number of the fluid $\frac{\epsilon}{\delta^{2}}=O(1)$, and this assumption means that the dispersion and nonlinearity are equally important. Then the system of Eq.(4.26) and Eq.(4.27) take the form:

$$
\begin{gathered}
\eta_{t^{*}}^{*}+2 \epsilon \eta_{x^{*}}^{*} u^{*}+\epsilon \eta^{*} u_{x^{*}}^{*}+u_{x^{*}}^{*}=0, \\
u_{t^{*}}^{*}+\eta_{x^{*}}^{*}+2 \epsilon u^{*} u_{x^{*}}^{*}-\delta^{2} d u_{x^{*} x^{*} t^{*}}^{*}=0,
\end{gathered}
$$

where $d=\frac{4 \alpha+1}{8}$. We rewrite the model omitting the * in the form:

$$
\begin{gathered}
\eta_{t}+2 \epsilon \eta_{x} u+\epsilon \eta u_{x}+u_{x}=0, \\
u_{t}+\eta_{x}+2 \epsilon u u_{x}-\delta^{2} d u_{x x t}=0,
\end{gathered}
$$

To solve the system, we make the assumption:

$$
u=\eta+\epsilon A+\delta^{2} B,
$$

where $A$ and $B$ are functions of $x$ and $t$. Therefore we have

$$
\begin{array}{r}
u_{x}=\eta_{x}+\epsilon A_{x}+\delta^{2} B_{x}, \\
u_{t}=\eta_{t}+\epsilon A_{t}+\delta^{2} B_{t}, \\
u_{x x t}=\eta_{x x t}+\epsilon A_{x x t}+\delta^{2} B_{x x t} .
\end{array}
$$

Substitute the relations above into Eq.(4.31) and Eq.(4.32), we have

$$
\begin{aligned}
& \eta_{t}+2 \epsilon \eta_{x} \eta+\eta_{x}+\epsilon A_{x}+\delta^{2} B_{x}+2 \eta \eta_{x}=O\left(\epsilon^{2}, \delta^{4}\right), \\
& \eta_{t}+\epsilon A_{t}+\delta^{2} B_{t}+2 \epsilon \eta \eta_{x}+\eta_{x}-\delta^{2} d \eta_{x x t}=O\left(\epsilon^{2} \delta^{4}\right) .
\end{aligned}
$$

In order to find nontrivial solutions for Eq.(4.34) and Eq.(4.35), we compare 
the coefficients of the equations, immediately we observe the relation :

$$
\begin{gathered}
A_{x}+3 \eta \eta_{x}=A_{t}+2 \eta \eta_{x}, \\
B_{x}=B_{t}-d \eta_{x x t}
\end{gathered}
$$

By Eq.(4.34) and Eq.(4.35), we know that

$$
u_{t}+u_{x}=O(\epsilon)
$$

thereby

$$
\eta_{t}+\eta_{x}=O\left(\epsilon, \delta^{2}\right) .
$$

Then we combine Eq.(4.33), one obtains

$$
\epsilon\left(A_{t}+A_{x}\right)+\delta^{2}\left(B_{t}+B_{x}\right)=O\left(\epsilon, \delta^{2}\right) .
$$

So we have that

$$
\begin{aligned}
& A_{t}+A_{x}=O\left(\epsilon, \delta^{2}\right), \\
& B_{t}+B_{x}=O\left(\epsilon, \delta^{2}\right) .
\end{aligned}
$$

Then we solve for Eq.(4.36) and Eq.(4.37), we have the following relation

$$
\begin{aligned}
A_{t} & =\frac{1}{2} \eta \eta_{x}, \\
B_{t} & =\frac{d}{2} \eta_{x x t} .
\end{aligned}
$$

Now we can subsitute $A_{t}$ and $B_{t}$ into Eq.(4.35). Hence we obtain the BBM equation for the system:

$$
\eta_{t}+\eta_{x}+\frac{5 \epsilon}{2} \eta \eta_{x}-\frac{d}{2} \delta^{2} \eta_{x x t}=0
$$

and the KdV equation.

$$
\eta_{t}+\eta_{x}+\frac{5 \epsilon}{2} \eta \eta_{x}+\frac{d}{2} \delta^{2} \eta_{x x x}=0
$$


Then we have the dimensional form of Eq.(4.38),

$$
\eta_{t}+\frac{1}{2} \sqrt{\frac{2 E h}{\rho r_{0}}} \eta+\frac{5}{4 r_{0}} \sqrt{\frac{2 E h}{\rho r_{0}}} \eta \eta_{x}-\frac{r_{0}^{2} d}{2} \frac{1}{2} \sqrt{\frac{2 E h}{\rho r_{0}}} \eta_{x x t}=0
$$

and the dimensional form of Eq.(4.39),

$$
\eta_{t}+\frac{1}{2} \sqrt{\frac{2 E h}{\rho r_{0}}} \eta+\frac{5}{4 r_{0}} \sqrt{\frac{2 E h}{\rho r_{0}}} \eta \eta_{x}+\frac{r_{0}^{2} d}{2} \frac{1}{2} \sqrt{\frac{2 E h}{\rho r_{0}}} \eta_{x x x}=0 .
$$

\subsection{Dissipative Boussinesq systems}

In order to obtain a more accurate system, now we can take the viscosity into account. Recall the Euler equations in the previous chapters:

$$
\begin{gathered}
u_{t}+\epsilon u u_{x}+\epsilon v u_{r}+p_{x}=0, \\
\delta^{2}\left[v_{t}+\epsilon u v_{x}+\epsilon v v_{r}\right]+p_{r}=0 \\
r u_{x}+(r v)_{r}=0 .
\end{gathered}
$$

Since we are going to take the viscosity into account, we need to use the Navier-Stokes equation, which can be written in cylinderical coordinates in the non-dimensional and scaled form:

$$
\begin{gathered}
u_{t}+\epsilon u u_{x}+\epsilon v u_{r}+p_{x}=\frac{1}{\delta^{2}} \frac{1}{R e}\left[\frac{1}{r}\left(r u_{r}\right)_{r}+\delta^{2} u_{x x}\right], \\
\delta^{2}\left[v_{t}+\epsilon u v_{x}+\epsilon v v_{r}\right]+p_{r}=\frac{1}{R e}\left[\frac{1}{r}\left(r v_{r}\right)_{r}-\frac{v}{r^{2}}+\delta^{2} v_{x x}\right], \\
r u_{x}+(r v)_{r}=0,
\end{gathered}
$$

where $R e$ is the Reynolds number and defined as:

$$
R e=\frac{\lambda \tilde{c}}{\kappa} .
$$

In the relation above, $\kappa$ denotes the kinematic viscosity and $\tilde{c}$ is a constant. We assume that the viscosity is very small, specifically we take: 


$$
\frac{1}{R e}=O\left(\epsilon \delta^{2}\right)
$$

After discarding the terms of $O\left(\epsilon, \delta^{2}\right)$, we have the following system:

$$
\begin{gathered}
u_{t}+\epsilon u u_{x}+\epsilon v u_{r}+p_{x}=\frac{1}{\delta^{2}} \frac{1}{R e} \frac{1}{r}\left(r u_{r}\right)_{r} \\
\delta^{2}\left[v_{t}+\epsilon u v_{x}+\epsilon v v_{r}\right]+p_{r}=0 \\
r u_{x}+(r v)_{r}=0
\end{gathered}
$$

Following some heuristic arguments [12], [20]. We apply a further substitution, taking

$$
u(x, r, t)=u^{w}(x, t) \phi(x, r, t)+O\left(\delta^{2}\right)
$$

and assume that

$$
\phi(x, r, t)=2 \frac{r^{w 2}-r^{2}}{r_{0}^{2}} .
$$

Thereby we can see that the viscous terms has the form as below:

$$
\frac{1}{r}\left(r u_{r}\right)_{r}=-8 \frac{u^{w}}{r_{0}^{2}}
$$

Hence we rewrite the system:

$$
\begin{gathered}
u_{t}+\epsilon u u_{x}+\epsilon v u_{r}+p_{x}=-\frac{1}{\delta^{2}} \frac{8}{R e} \frac{u^{w}}{r_{0}^{2}}, \\
\delta^{2}\left[v_{t}+\epsilon u v_{x}+\epsilon v v_{r}\right]+p_{r}=0, \\
r u_{x}+(r v)_{r}=0 .
\end{gathered}
$$

Following the same derivation as in Chapter 3, we obtain the Boussi- 
nesq system:

$$
\begin{gathered}
u_{t}+\epsilon u u_{x}+(\beta(x) \eta)_{x}-\alpha \delta^{2}\left(\frac{r^{0}}{2} u_{x}\right)_{x t}-\alpha \delta^{2}\left(r_{0 x} u\right)_{x t} \\
-\delta^{2} u_{x t} \frac{r_{0} r_{0 x}}{2}-\frac{1}{\delta^{2}} \frac{8}{R e} \frac{u^{w}}{r_{0}^{2}}=0, \\
\eta_{t}+r_{0 x} u+\epsilon \eta_{x} u+\frac{r_{0}}{2} u_{x}+\frac{\epsilon \eta}{2} u_{x}+\delta^{2} u_{x x} \frac{\left(r_{0}\right)^{2} r_{0 x}}{4}+\delta^{2} u_{x x x} \frac{\left(r_{0}\right)^{3}}{16}=0 .
\end{gathered}
$$

The system of Eq.(4.54) and Eq.(4.55) can be written in the dimensional form:

$$
\begin{gathered}
u_{t}+u u_{x}+\left(\frac{E_{\sigma}(x) h}{r_{0}^{2} \rho} \eta\right)_{x}-\frac{\rho^{w} h}{\rho}\left(\frac{r_{0}}{2} u_{x}\right)_{x t}-\frac{\rho^{w} h}{\rho}\left(r_{0 x} u\right)_{x t} \\
-u_{x t} \frac{r_{0} r_{0 x}}{2}+8 \kappa \frac{u^{w}}{r_{0}^{2}}=0, \\
\eta_{t}+r_{0 x} u+\eta_{x} u+\frac{r_{0}}{2} u_{x}+\frac{\eta}{2} u_{x}+u_{x x} \frac{\left(r_{0}\right)^{2} r_{0 x}}{4}+u_{x x x} \frac{\left(r_{0}\right)^{3}}{16}=0 .
\end{gathered}
$$

Recall that Eq.(4.14) and Eq.(4.15) form the simplest system among all the systems in this chapter, we can also take dissipative terms for them:

$$
\begin{gathered}
\eta_{t}+\frac{1}{2}\left(r_{0}+\eta\right) u_{x}^{\theta}+\left(r_{0 x}+\eta_{x}\right) u^{\theta}=0, \\
\left(1-\bar{\alpha} r_{0 x x t}\right) u_{t}^{\theta}+[\beta \overline{(x)} \eta]_{x}+u^{\theta} u_{x}^{\theta}-\frac{\left(3 \alpha+r_{0}\right) r_{0 x}}{2} u_{x t}^{\theta} \\
-\frac{\left(4 \bar{\alpha}+r_{0}\right) r_{0}}{8} u_{x x t}^{\theta}+8 \kappa \frac{u^{\theta}}{r_{0}^{2}}=0,
\end{gathered}
$$

which will be convenient for some numerical computation to test the model. 


\section{Chapter 5}

\section{Properties of the general system}

Some important properties of the general system are being studied here. We study the dispersion relation of the general system and that of the Euler equations. We study the well-posedness of the linearized systems and weakly non-linear systems. Then we invesigate the existence of solitary wave solutions.

\subsection{Dispersion relation for the Euler equations}

Now we consider the dispersion relation of the Euler system in this study. First we linearise the non-dimensional Euler system as below

$$
\begin{gathered}
u_{t}+\frac{p_{x}}{\rho}=0, \\
v_{t}+\frac{p_{r}}{\rho}=0, \\
u_{x}+v_{r}+\frac{v}{r}=0 .
\end{gathered}
$$

And for $r=r^{w}$, we have

$$
u=\eta_{t}
$$




$$
\rho^{w} h \eta_{t t}=p-\frac{E h}{r_{0}^{2}} \eta .
$$

Additionally, we have that $v=0$ when $r=0$. Suppose the variables has the following relations

$$
\begin{aligned}
& u=u_{0}(r) e^{i(k x-\omega t)}, \\
& v=v_{0}(r) e^{i(k x-\omega t),} \\
& p=p_{0}(r) e^{i(k x-\omega t)}, \\
& \eta=\eta_{0} e^{i(k x-\omega t),}
\end{aligned}
$$

where $u_{0}(r), v_{0}(r)$ and $p_{0}(r)$ are function or $r$ and $\eta_{0}$ is constant.

From Eq.(5.1), we have

$$
u_{t} r+\frac{p_{x} r}{\rho}=0 .
$$

For Eq.(5.2), we have

$$
v_{t} x+\frac{p_{r} x}{\rho}=0,
$$

For Eq.(5.1), we obtain

$$
p_{0}=\frac{\omega}{k} u_{0}
$$

By Eq.(5.7) and Eq.(5.9), and consider the relation in Eq.(5.6), we have

$$
i k v_{0}=u_{0 r}
$$

So we have that

$$
i k v_{0 r}=u_{0 r r} .
$$

Then we substitute Eq.(5.11) into Eq.(5.3), one obatin

$$
r u_{0 r r}+u_{0 r}-r k^{2} u_{0}=0
$$


which is the Bessel equation. Since we have

$$
v(0)=0, v=v_{0}(r) e^{i(k x-\omega t)}
$$

we conclude that

$$
u_{0 r}=0 .
$$

Similarly, we have that $u_{0 r}=\omega k \eta_{0}$ by Eq.(5.4). Combine Eq(5.13) and the knowledge of the Bessel function, one obtains

$$
u_{0}(r)=\frac{\eta_{0} \omega I_{0}(k r)}{I_{r}\left(k r_{0}\right)}
$$

where $I_{0}$ and $I_{1}$ are Bessel functions.Now we consider the boundary condition when $r=r_{0}$, By substituting Eq.(5.9) and Eq.(5.14) into Eq(5.5), we obtains the relation between $\omega$ and $\mathrm{k}$,

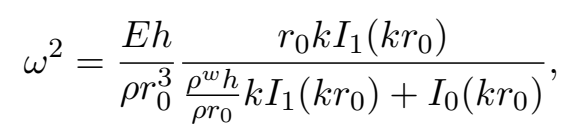

For convenience, we denote the dispersion relation for the Euler system by $\omega_{\epsilon}$. Now we study the phase velocity of the Euler system, and consider that the system is under the long-wave condition, which means that $k$ is very small. Then we can use the Taylor series to expand the phase velocity. Since the phase velocity is defined as:

$$
c(k)=\frac{\omega(k)}{k},
$$

We obtain the following expansion:

$$
\begin{array}{r}
\frac{c_{\epsilon}(k)}{c_{0}}=1-\frac{4 \alpha+1}{2^{4}}\left(r_{0} k\right)^{2}+\frac{144 \alpha^{2}+72 \alpha+13}{3 \times 2^{9}}\left(r_{0} k\right)^{4} \\
-\frac{960 \alpha^{3}+720 \alpha^{2}+228 \alpha+31}{3 \times 2^{13}}\left(r_{0} k\right)^{6} \\
+\frac{403200 \times \alpha^{4}+403200 \alpha^{3}+180000 \alpha^{2}+42480 \alpha+4591}{5 \times 3 \times 2^{19}}\left(r_{0} k\right)^{8} \\
+O\left(k^{10}\right),
\end{array}
$$


where $c_{0}$ is the Moens-Korteweg characteristic velocity, which is defined as:

$$
c_{0}=\sqrt{\frac{E h}{2 \rho r_{0}}} .
$$

\subsection{Dispersion relation of the Boussinesq system}

Similar with what we have done in Chapter 3, we can also compute the dispersion relation of the general system with constant radius. We take that

$$
u^{\theta}=u_{0} e^{i(k x-\omega t)}, \quad \eta^{\theta}=\eta_{0} e^{i(k x-\omega t)},
$$

and then we consider the linearised system of Eq.(4.24) and Eq.(4.25):

$$
\begin{gathered}
\eta_{t}+\frac{1}{2} r_{0} u_{x}^{\theta}+r_{0}^{3} a u_{x x x}^{\theta}-r_{0}^{2} b \eta_{x x t}=0, \\
u_{t}^{\theta}+\frac{E h}{\rho r_{0}^{2}} \eta_{x}+\frac{E h}{\rho r_{0}^{2}} r_{0}^{2} c \eta_{x x x}-r_{0}^{2} d u_{x x t}^{\theta}=0 .
\end{gathered}
$$

Thus we can compute the dispersion relation,

$$
\omega^{2}(k)=\frac{E h}{\rho r_{0}^{3}} \frac{\left(1-c\left(r_{0} k\right)^{2}\right)\left(\frac{1}{2}-a\left(r_{0} k\right)^{2}\right)}{\left(1+b\left(r_{0} k\right)^{2}\right)\left(1+d\left(r_{0} k\right)^{2}\right)}\left(r_{0} k\right)^{2} .
$$

Denoting the $\omega$ for the Boussinesq system by $\omega_{\mathcal{B}}$, we compare it with the dispersion relation of the Euler equations, which has been computed in Section 5.1:

$$
\omega_{\epsilon}^{2}(k)=\frac{E h}{\rho r_{0}^{3}} \frac{k I_{1}\left(k r_{0}\right)}{\frac{\rho^{2} h}{\rho r_{0}} k I_{1}\left(k r_{0}\right)+I_{0}\left(k r_{0}\right)},
$$


which has the Taylor expansion as:

$$
\begin{array}{r}
\frac{c_{\epsilon}(k)}{c_{0}}=1-\frac{4 \alpha+1}{2^{4}}\left(r_{0} k\right)^{2}+\frac{144 \alpha^{2}+72 \alpha+13}{3 \times 2^{9}}\left(r_{0} k\right)^{4} \\
-\frac{960 \alpha^{3}+720 \alpha^{2}+228 \alpha+31}{3 \times 2^{13}}\left(r_{0} k\right)^{6} \\
+\frac{403200 \times \alpha^{4}+403200 \alpha^{3}+180000 \alpha^{2}+42480 \alpha+4591}{5 \times 3 \times 2^{19}}\left(r_{0} k\right)^{8} \\
+O\left(k^{10}\right) .
\end{array}
$$

We can also take the Taylor expansion for the Boussinesq system:

$$
\begin{array}{r}
\frac{c_{\mathcal{B}}(k)}{c_{0}}=1-\frac{4 \alpha+1}{2^{4}}\left(r_{0} k\right)^{2} \\
-\frac{1}{2^{9}}[64 \mu-48) \alpha^{2}+\left(64 \mu\left(1-\theta^{2}\right)+32 \theta^{2}-40\right) \alpha \\
\left.+16(\mu+\nu-1) \theta^{4}-8(4 \mu+2 \nu-3) \theta^{2}+16 \mu+4 \nu-11\right]\left(r_{0} k\right)^{4} \\
-\frac{\mathcal{P}_{3}}{2^{13}}\left(r_{0} k\right)^{6}+\frac{\mathcal{P}_{4}}{2^{19}}\left(r_{0} k\right)^{8}+O\left(k^{10}\right),
\end{array}
$$

where $\mathcal{P}_{3}$ and $\mathcal{P}_{4}$ are polynomials of $\alpha, \mu, \nu$, and $\theta^{2}$. Observe that the first two terms of the Euler system and all Boussinesq system are the same, and the differences between them can be reduced by choosing different $\mu, n u$ and $\theta$. Then we can take $\theta^{2}=1 / 2$ and $\mu=\nu=0$ for simplification and take only the low order terms of Eq.(5.20), which leads:

$$
\frac{c_{\mathcal{B}}(k)}{c_{0}}=1-\frac{4 \alpha+1}{2^{4}}\left(r_{0} k\right)^{2}+\frac{144 \alpha^{2}+72 \alpha+9}{3 \times 2^{9}}\left(r_{0} k\right)^{4} .
$$

Then terms of Eq.(5.21) is very similar the low order terms of the Euler dispersion relation. Observe that the terms of $k^{2}$ are the same and we only have a small difference in the term of $k^{4}$. Now we consider the realistic data [23] in Table 5.1:

The graph with two dispersion relations is presented in Figure 5.1, where the red curve represents the Euler equations and green curve represents the Boussinesq system. 


\begin{tabular}{ccc}
\hline Parameter & corresponding letter & \\
\hline Vessel's length & $L$ & $1.26 \times 10^{-2} \mathrm{~m}$ \\
Vessel's nominal radius & $R$ & $3 \times 10^{-3} \mathrm{~m}$ \\
Vessel's thickness & $h$ & $3 \times 10^{-4} \mathrm{~m}$ \\
Wall density & $\rho^{w}$ & $1000 \mathrm{~kg} / \mathrm{m}^{3}$ \\
Blood density & $\rho$ & $1060 \mathrm{~kg} / \mathrm{m}^{3}$ \\
Young's modulus & $E$ & $4.07 \times 10^{5} \mathrm{~kg} /\left(\mathrm{msec}^{2}\right)$ \\
Kinetic viscosity & $\kappa$ & $4 \times 10^{-6} \mathrm{~m} / \mathrm{sec}$ \\
\hline
\end{tabular}

Table 5.1: Parameters in the numerical experiments

\subsection{Linear well-posedness of the Boussinesq system}

In order to study the well-posedness of the system, first we consider the general linearised system

$$
\begin{gathered}
\eta_{t}+\frac{1}{2} u_{x}^{\theta}+\delta^{2} a u_{x x x}^{\theta}-\delta^{2} b \eta_{x x t}=0, \\
u_{t}^{\theta}+\beta \eta_{x}+\delta^{2} \beta c \eta_{x x x}-\delta^{2} d u_{x x t}^{\theta}=0,
\end{gathered}
$$

where $a, b, c$ and $d$ is defined as in the previous chapter:

$$
\begin{aligned}
& a=\frac{\left(2 \theta^{2}-1\right) \nu}{16}, \quad b=\frac{\left(2 \theta^{2}-1\right)(1-\nu)}{8}, \\
& c=\frac{\left(2 \alpha+\left(1-\theta^{2}\right)\right) \mu}{4}, \quad d=\frac{\left(2 \alpha+\left(1-\theta^{2}\right)\right)(1-\mu)}{4} .
\end{aligned}
$$

We denote the $L_{p}(\mathbb{R})$ norm of a function $f$ by $|f|_{p}$, and the norm of the Sobolev space $H^{s}(\mathbb{R})$ by $\|f\|_{s}$. For a norm on $H^{s}$, we have

$$
\|f\|_{s}^{2}=\Sigma_{i=1}^{s}\left|f^{(i)}\right|_{2}^{2}
$$

And if $s$ is not an integer, we take

$$
\|f\|_{s}^{2}=\frac{1}{2 \pi} \int_{-\infty}^{+\infty}|\hat{f}(k)|^{2}\left(1+k^{2}\right)^{s} d k
$$




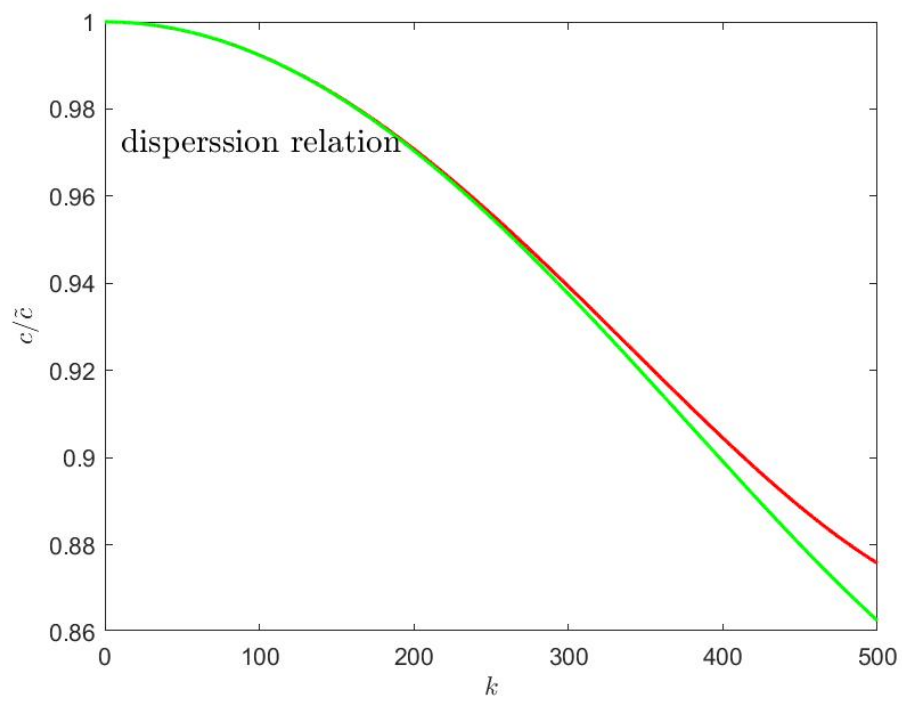

Figure 5.1: Dispersion relation (Euler in red and Boussinesq in green)

Now we are going to consider the initial value problem with

$$
\eta_{0}=\phi, \quad u_{0}=\psi
$$

Both $\phi$ and $\psi$ tend to 0 as $x$ goes to positive or negative infinity. Then we take the Fourier transform with respect to $x$, which leads to:

$$
\frac{d}{d t}\left(\begin{array}{c}
\hat{\eta} \\
\hat{u}
\end{array}\right)+i k A(k)\left(\begin{array}{c}
\hat{\phi} \\
\hat{\psi}
\end{array}\right)=0
$$

where

$$
\begin{gathered}
A(k)=\left(\begin{array}{cc}
0 & \omega_{1}(k) \\
\omega_{2}(k) & 0
\end{array}\right), \\
\omega_{1}=\frac{1}{2} \frac{1-2 \delta^{2} a k^{2}}{1+\delta^{2} b k^{2}}, \quad \omega_{2}=\frac{1-\delta^{2} \beta c k^{2}}{1+\delta^{2} d k^{2}} .
\end{gathered}
$$

Thereby we know that for the initial value of $\eta_{0}$ and $u_{0}$, we can solve for Eq. (5.26), and obtain: 


$$
\left(\begin{array}{c}
\hat{\eta} \\
\hat{u}
\end{array}\right)=e^{-i k A(k) t}\left(\begin{array}{c}
\hat{\eta_{0}} \\
\hat{u_{0}}
\end{array}\right)
$$

We can diagonalize the matrix $A(k)$ as:

$$
A(k)=P B P^{-1},
$$

where $P$ is an invertible matrix and $A$ is a diagonal matrix. Then we have that

$$
\begin{gathered}
P(k)=\left(\begin{array}{cc}
-\sqrt{\frac{\omega_{1}}{\omega_{2}}} & \sqrt{\frac{\omega_{1}}{\omega_{2}}} \\
1 & 1
\end{array}\right), \\
B(k)=\left(\begin{array}{cc}
-\sqrt{\omega_{1} \omega_{2}} & 0 \\
0) & \sqrt{\omega_{1} \omega_{2}}
\end{array}\right),
\end{gathered}
$$

Now we take

$$
\sigma(k)=\left|\omega_{1}(k) \omega_{2}(k)\right|^{\frac{1}{2}}
$$

Since

$$
e^{P A P^{-1}}=P e^{A} P^{-1}
$$

then for $\omega_{1}(k) \omega_{2}(k) \geq 0$, we have

$$
e^{-i k A(k) t}=\left(\begin{array}{cc}
\cos (k \sigma(k) t) & -i \sin (k \sigma(k) t) \frac{\omega_{1}(k)}{\sigma(k)} \\
i \sin (k \sigma(k) t) \frac{\omega_{1}(k)}{\sigma(k)} & \cos (k \sigma(k) t) .
\end{array}\right)
$$

When $\omega_{1}(k) \omega_{2}(k) \leq 0$, we have

$$
e^{-i k A(k) t}=\left(\begin{array}{cc}
\cosh (k \sigma(k) t) & -i \sinh (k \sigma(k) t) \frac{\omega_{1}(k)}{\sigma(k)} \\
i \sinh (k \sigma(k) t) \frac{\omega_{1}(k)}{\sigma(k)} & \cosh (k \sigma(k) t) .
\end{array}\right)
$$

By the theory of Fourier multipliers [3], we know that the system is wellposed if the matrix $e^{-i k A(k) t}$ is bounded in finite intervals of $t$. Thus for each combination of the parameters the rational function $\frac{\omega_{1}}{\omega_{2}}$ has neither zeros nor poles on the real axis. The exact form of $\omega_{1} / \omega_{2}$ is: 


$$
\frac{\omega_{1}}{\omega_{2}}=\frac{1}{2} \frac{1-2 \delta^{2} a k^{2}}{1+\delta^{2} b k^{2}} \frac{1+\delta^{2} d k^{2}}{1-\delta^{2} \beta c k^{2}} .
$$

Obviously we have that $\beta>0$, so we only need to analyze if $a, b, c$ and $d$ are positive, negative or zero. Here we have four factors, $1-2 \delta^{2} a k^{2}, 1+\delta^{2} b k^{2}$, $1+\delta^{2} d k^{2}$ and $1-\delta^{2} \beta c k^{2}$. Since we don't want the result to go across 0 , and obviously for some $k$ all factors have positive value, we need the factors to be "properly" positive. First we can assume that all factors are positive, then the ratio is always positive. Then we can consider the case when some negative factors can cancelled with some other factors. Hence we know that one of the following conditions must hold:

$$
\begin{gathered}
(C 1) \quad b \geq 0, d \geq 0, a \leq 0, c \leq 0, \\
(C 2) \quad b \geq 0, d \geq 0,2 a=\beta c>0, \\
(C 3) \quad b=d<0,2 a=\beta c>0 .
\end{gathered}
$$

By Eq.(5.27) and Eq.(5.28), we can obtain that

$$
|\hat{\eta}|^{2}+\left(\frac{\omega_{1}}{\omega_{2}}\right)|\hat{u}|^{2}=|\hat{\phi}|^{2}+\left(\frac{\omega_{1}}{\omega_{2}}\right)|\hat{\psi}|^{2} .
$$

Here we can define the Fourier multiplier operator $\mathcal{H}$ as following:

$$
\hat{\mathcal{H}} g(k)=h(k) \hat{g}(k)
$$

where

$$
h(k)=\left(\frac{\omega_{1}}{\omega_{2}}\right)^{1 / 2} .
$$

Then the "energy" for any $s$ of the solution is, [4] :

$$
\|\eta\|^{2}+\|\mathcal{H} u\|^{2}=\|\phi\|^{2}+\|\mathcal{H} \psi\|^{2} .
$$

By Eq.(5.25), we know that the operator $\mathcal{H}$ raise the order of the corresponding variable by $l$. Then we obtain the following :

Theorem 5.1 Let $a, b, c, d$ satisfy (C1)-(C3). Define the order $l$ pseudodifferential operator $\mathcal{H}$ as above and set $m_{1}=\max (0,-l), m_{2}=\max (0, l)$. 
Then the corresponding linear initial-value problem is well posed in $H^{s+m_{1}} \times$ $H^{s+m_{2}}$ for any $s \geq 0$.

For example, if $(C 1)$ is satisfied, obviously that $\frac{\omega_{1}}{\omega_{2}}$ is always positive. In either of the above conditions implies that $\omega_{1} \omega_{2} \leq 0$. Now we investigate the linearised system $\mathrm{Eq}(5.17)$ and $\mathrm{Eq}(5.18)$.

$$
\begin{gathered}
\eta_{t}+\frac{1}{2} u_{x}^{\theta}+\delta^{2} a u_{x x x}^{\theta}-\delta^{2} b \eta_{x x t}=0, \\
u_{t}^{\theta}+\beta \eta_{x}+\delta^{2} \beta c \eta_{x x x}-\delta^{2} d u_{x x t}^{\theta}=0,
\end{gathered}
$$

(i) For a classical Boussinesq system, we have

$$
\theta^{2}=\frac{1}{2}, \quad \nu \text { is abitrary, } \mu=0 \text {. }
$$

Then we know that

$$
a=b=c=0, \quad d=\frac{4 \alpha+1}{8} .
$$

Hence it satisfies condition $(C 1)$.

(ii) For a BBM-BBM type system, we have

$$
\mu=\nu=0
$$

thus

$$
a=0, \quad b=\frac{2 \theta^{2}-1}{8}, \quad c=0, \quad d=\frac{2 \alpha+\left(1-\theta^{2}\right)}{4} .
$$

Since $a=c=0$, it only can be $C(1)$ if the system is well-posed. Thus we need $\theta$ satisfies that:

$$
\theta^{2} \leq \frac{1}{2}, \quad \theta^{2} \leq 2 \alpha+1
$$

(iii) For a KdV-BBM system, we have

$$
\nu=1, \quad \mu=0,
$$

thus

$$
a=\frac{2 \theta^{2}-1}{16}, \quad b=c=0, \quad d=\frac{2 \alpha+\left(1-\theta^{2}\right)}{4} .
$$


Then it can be seen if $a$ is not 0 , the system is not well-posed.

(iv) For a KdV-KdV system, we have

$$
\nu=1, \quad \mu=1,
$$

thus

$$
b=0, \quad a=\frac{2 \theta^{2}-1}{8}, \quad c=\frac{2 \alpha+1-\theta^{2}}{4}, \quad d=0 .
$$

Observe that $c>0$ the situation is similar to (ii), hence we have if

$$
\theta^{2}=\frac{2 \alpha \beta+\beta+1}{\beta+2}
$$

the system is well-posed.

(v) For a Bona-Smith type system, we have

$$
\nu=0, \quad \mu \text { is arbitray provided } 2\left(\alpha+\left(1-\theta^{2}\right)\right) \mu<0 .
$$

Therefore only $(C 1)$ can be satisfied if the equation is well-posed. Then we know that $b \geq 0, \quad d \geq 0$. Since $\left(\alpha+\left(1-\theta^{2}\right)\right)>0$, we know that $\mu<0$. Hence we have when

$$
\theta^{2} \geq \frac{1}{2}
$$

the system is well-posed.

\subsection{Well-possedness of the non-linear system}

Here we consider the local existence and uniqueness of solution of the non-linear system:

$$
\begin{gathered}
\eta_{t}+\frac{1}{2}(1+\epsilon \eta) u_{x}^{\theta}+\epsilon \eta_{x} u^{\theta}+\delta^{2} a u_{x x x}^{\theta}-\delta^{2} b \eta_{x x t}=0, \\
u_{t}^{\theta}+\beta \eta_{x}+\epsilon u^{\theta} u_{x}^{\theta}+\delta^{2} \beta c \eta_{x x x}-\delta^{2} d u_{x x t}^{\theta}=0
\end{gathered}
$$

with [3]:

$$
b>0, \quad d>0 .
$$


Then we take Fourier transform with respect to $x$ :

$$
\frac{d}{d t}\left(\begin{array}{c}
\hat{\eta} \\
\hat{u}
\end{array}\right)+i k A(k)\left(\begin{array}{c}
\hat{\eta} \\
\hat{u}
\end{array}\right)=-i k P^{-1}\left(\begin{array}{c}
\frac{1}{2} \frac{1}{\left.1+\frac{\delta^{2} b k^{2}}{\left(\overrightarrow{u \eta_{x}}\right.}+\frac{1}{2} \widehat{u_{x} \eta}\right)} \\
\frac{1}{2} \frac{1}{1+\delta^{2} d k^{2}} \widehat{u^{2}}
\end{array}\right)
$$

where

$$
\begin{gathered}
A(k)=\left(\begin{array}{cc}
0 & \omega_{1}(k) \\
\omega_{2}(k) & 0
\end{array}\right), \\
\omega_{1}=\frac{1}{2} \frac{1-2 \delta^{2} a k^{2}}{1+\delta^{2} b k^{2}}, \quad \omega_{2}=\frac{1-\delta^{2} \beta c k^{2}}{1+\delta^{2} d k^{2}},
\end{gathered}
$$

which is the same as in the previous section. In order to study the wellpossedness of the system, we take the change of variables as below:

$$
\begin{gathered}
\eta=\mathcal{H}(v+w), \\
u=v-w,
\end{gathered}
$$

where $\mathcal{H}$ is the Fourier multiplier, which satisfies:

$$
\widehat{\mathcal{H} g}=h(k) \hat{g}(k), \quad h(k)=\left(\frac{\omega_{1}(k)}{\omega_{2}(k)}\right)^{\frac{1}{2}} .
$$

Then Eq.(5.34) will have the form:

$$
\begin{gathered}
\frac{d}{d t}\left(\begin{array}{c}
\hat{v} \\
\hat{w}
\end{array}\right)+i k\left(\begin{array}{cc}
\sigma(k) & 0 \\
0 & -\sigma(k)
\end{array}\right)\left(\begin{array}{c}
\hat{v} \\
\hat{w}
\end{array}\right)= \\
-i k P^{-1}\left(\begin{array}{c}
\frac{1}{2} \frac{1}{\left.1+\frac{\delta^{2} b k^{2}}{\left(\widehat{u \eta_{x}}\right.}+\frac{1}{2} \widehat{u_{x} \eta}\right)} \\
\frac{1}{2} \frac{1}{1+\delta^{2} d k^{2}} \widehat{u^{2}}
\end{array}\right),
\end{gathered}
$$

where

$$
\sigma(k)=\left(\omega_{1}(k) \omega_{2}(k)\right)^{\frac{1}{2}}, \quad P^{-1}=\frac{1}{2}\left(\begin{array}{cc}
\frac{1}{h(k)} & 1 \\
\frac{1}{h(k)} & -1
\end{array}\right) .
$$


For Eq.(5.35), we consider $\mathcal{H}$ of different order.

Consider first $\mathcal{H}$ is of order 0 . Here we also need to apply the condition when the system is linearly well-posed, which means that one of the following conditions holds:

$$
\begin{array}{rrr}
a<0, \quad c<0, \quad b>0, & , d>0 \\
\text { or } \quad a=c>0, \quad b>0, & d>0 .
\end{array}
$$

At the beginning we consider the equivalent system Eq.(5.35) completed with initial values $v_{0}, w_{0}$, where

$$
\begin{aligned}
& v_{0}=\frac{\mathcal{H}^{-1}(\phi)+\psi}{2} \in H^{s}(\mathbb{R}), \\
& w_{0}=\frac{\mathcal{H}^{-1}(\phi)-\psi}{2} \in H^{s}(\mathbb{R}),
\end{aligned}
$$

Then we take the inverse Fourier transform for Eq.(5.35), it follows that

$$
\frac{\partial}{\partial t}\left(\begin{array}{c}
v \\
w
\end{array}\right)+\mathcal{B}\left(\begin{array}{c}
v \\
w
\end{array}\right)=\mathcal{F}\left(\begin{array}{c}
v \\
w
\end{array}\right)
$$

where

$$
\begin{gathered}
\mathcal{B}=\left(\begin{array}{cc}
\sigma(k) & 0 \\
0 & -\sigma(k)
\end{array}\right), \\
\mathcal{F}\left(\begin{array}{c}
v \\
w
\end{array}\right)=-P^{-1}\left(\begin{array}{c}
\left(I-\delta^{2} b \partial_{x}^{2}\right)^{-1}\left[\frac{1}{2} \mathcal{H}(v+w) \partial_{x}(v-w)+\partial_{x} \mathcal{H}(v+w)(v-w)\right] \\
\left(I-\delta^{2} d \partial_{x}^{2}\right)^{-1}(v-w) \partial_{x}(v-w)
\end{array}\right),
\end{gathered}
$$

$I$ is the identity operator and $\left(I-\delta^{2} b \partial_{x}^{2}\right)^{-1}$ is the inverse operator of $(I-$ $\left.\delta^{2} b \partial_{x}^{2}\right)$. If $S(t)$ denotes the group generated by $\mathcal{B}$, then we know that it is a unitary group on $H^{s}\left(\mathbb{R}^{2}\right)$ [3]. By Duhamel's formula, Eq.(5.37) also can be written in the following form:

$$
\left(\begin{array}{c}
v \\
w
\end{array}\right)=S(t)\left(\begin{array}{c}
v_{0} \\
w_{0}
\end{array}\right)+\int_{0}^{t} S(t-s) \mathcal{F}\left(\begin{array}{c}
v \\
w
\end{array}\right) d s .
$$

Now we consider some $\left(f_{1}, g_{1}\right)$ and $\left(f_{2}, g_{2}\right)$ in a closed ball of radius $R$ centred at $\mathbf{0}$, which means that 


$$
\begin{gathered}
\left\|f_{1}\right\|_{s}^{2}+\left\|g_{1}\right\|_{s}^{2} \leq R^{2}, \\
\left\|f_{2}\right\|_{s}^{2}+\left\|g_{2}\right\|_{s}^{2} \leq R^{2} .
\end{gathered}
$$

Let $A=\left(1-\delta^{2} b \partial_{x}^{2}\right)^{-1}$, we have

$$
\left\|\mathcal{F}\left(\begin{array}{l}
f_{1} \\
g_{1}
\end{array}\right)-\mathcal{F}\left(\begin{array}{l}
f_{2} \\
g_{2}
\end{array}\right)\right\|=\left\|P^{-1}\left(\begin{array}{c}
L_{1} \\
L_{2}
\end{array}\right)\right\|,
$$

where

$$
\begin{aligned}
& L_{1}= A\left[\frac{1}{2} \mathcal{H}\left(f_{1}+g_{1}\right) \partial_{x}\left(f_{1}-g_{1}\right)+\partial_{x} H\left(f_{1}+g_{1}\right)\left(f_{1}-g_{1}\right)\right. \\
&-\left.\frac{1}{2} \mathcal{H}\left(f_{2}+g_{2}\right) \partial_{x}\left(f_{2}-g_{2}\right)+\partial_{x} H\left(f_{2}+g_{2}\right)\left(f_{2}-g_{2}\right)\right], \\
& L_{2}=A\left[\left(f_{1}-g_{1}\right) \partial_{x}\left(f_{1}-g_{1}\right)-\left(f_{2}-g_{2}\right) \partial_{x}\left(f_{2}-g_{2}\right)\right] .
\end{aligned}
$$

Consider that $P^{-1}$ is bounded, we have

$$
\left\|\mathcal{F}\left(\begin{array}{l}
f_{1} \\
g_{1}
\end{array}\right)-\mathcal{F}\left(\begin{array}{c}
f_{2} \\
g_{2}
\end{array}\right)\right\| \leq C_{1}\left\|\left(\begin{array}{c}
L_{1} \\
L_{2}
\end{array}\right)\right\|,
$$

where $C_{1}$ is a constant. For a function $\mathbf{f}=\left(f_{1}, f_{2}\right)$ in a product space $X \times X$, the norm is defined as

$$
\|\mathbf{f}\|_{s}^{2}=\left\|f_{1}\right\|_{s}^{2}+\left\|f_{2}\right\|_{s}^{2}
$$

Then we have that

$$
\left\|\mathcal{F}\left(\begin{array}{l}
f_{1} \\
g_{1}
\end{array}\right)-\mathcal{F}\left(\begin{array}{c}
f_{2} \\
g_{2}
\end{array}\right)\right\| \leq C_{1}\left(\left\|L_{1}\right\|_{s}^{2}+\left\|L_{2}\right\|_{s}^{2}\right) .
$$

By the definition of $A$, we have

$$
\begin{aligned}
\left\|L_{1}\right\|_{s} & \leq C_{2} \| \frac{1}{2} \mathcal{H}\left(f_{1}+g_{1}\right) \partial_{x}\left(f_{1}-g_{1}\right)+\partial_{x} \mathcal{H}\left(f_{1}+g_{1}\right)\left(f_{1}-g_{1}\right) \\
& -\frac{1}{2} \mathcal{H}\left(f_{2}+g_{2}\right) \partial_{x}\left(f_{2}-g_{2}\right)+\partial_{x} \mathcal{H}\left(f_{2}+g_{2}\right)\left(f_{2}-g_{2}\right) \|_{s-2}^{2},
\end{aligned}
$$


and

$$
\left\|L_{2}\right\|_{s} \leq C_{3}\left\|\left(f_{1}-g_{1}\right) \partial_{x}\left(f_{1}-g_{1}\right)-\left(f_{2}-g_{2}\right) \partial_{x}\left(f_{2}-g_{2}\right)\right\|_{s-2}^{2},
$$

where $C_{i}$ denote constants for $i=1,2,3 \ldots$ Since for a norm we have

$$
\|a+b\|^{2} \leq C_{4}\left(\|a\|^{2}+\|b\|^{2}\right)
$$

thus we obtain that

$$
\begin{aligned}
\left\|L_{1}\right\|_{s} \leq C_{5}\left(\| \frac{1}{2} \mathcal{H}\left(f_{1}+g_{1}\right) \partial_{x}\left(f_{1}-g_{1}\right)-\frac{1}{2} \mathcal{H}\left(f_{2}+g_{2}\right) \partial_{x}\left(f_{2}-g_{2}\right)\right) \|_{s-2}^{2} \\
\left.+\left\|\partial_{x} \mathcal{H}\left(f_{1}+g_{1}\right)\left(f_{1}-g_{1}\right)-\partial_{x} \mathcal{H}\left(f_{2}+g_{2}\right)\left(f_{2}-g_{2}\right)\right\|_{s-2}^{2}\right) .
\end{aligned}
$$

Then we consider the substitution

$$
\begin{array}{r}
\frac{1}{2} \mathcal{H}\left(f_{1}+g_{1}\right) \partial_{x}\left(f_{1}-g_{1}\right)-\frac{1}{2} \mathcal{H}\left(f_{2}+g_{2}\right) \partial_{x}\left(f_{2}-g_{2}\right) \\
=\frac{1}{2} \mathcal{H}\left(f_{1}+g_{1}\right) \partial_{x}\left(f_{1}-g_{1}\right)-\frac{1}{2} \mathcal{H}\left(f_{1}+g_{1}\right) \partial_{x}\left(f_{2} g_{2}\right) \\
+\frac{1}{2} \mathcal{H}\left(f_{1}+g_{1}\right) \partial_{x}\left(f_{2} g_{2}\right)-\frac{1}{2} \mathcal{H}\left(f_{2}+g_{2}\right) \partial_{x}\left(f_{2}-g_{2}\right) \\
=\frac{1}{2} \mathcal{H}\left(f_{1}+g_{1}\right) \partial_{x}\left[\left(f_{1}-g_{1}\right)-\left(f_{2}-g_{2}\right)\right] \\
+\frac{1}{2} \mathcal{H}\left[\left(f_{1}-g_{1}\right)-\left(f_{2}-g_{2}\right)\right] \partial_{x}\left(f_{2}-g_{2}\right), \\
\partial_{x} \mathcal{H}\left(f_{1}+g_{1}\right)\left(f_{1}-g_{1}\right)-\partial_{x} \mathcal{H}\left(f_{2}+g_{2}\right)\left(f_{2}-g_{2}\right) \\
=\partial_{x} \mathcal{H}\left(f_{1}+g_{1}\right)\left(f_{1}-g_{1}\right)-\partial_{x} \mathcal{H}\left(f_{1}+g_{1}\right)\left(f_{1}-g_{1}\right) \\
-\partial_{x} \mathcal{H}\left(f_{1}+g_{2}\right)\left(f_{1}-g_{2}\right)-\partial_{x} \mathcal{H}\left(f_{2}+g_{2}\right)\left(f_{2}-g_{2}\right) \\
=\partial_{x} \mathcal{H}\left(f_{1}+g_{1}\right)\left[\left(f_{1}-g_{1}\right)-\left(f_{2}-g_{2}\right)\right] \\
-\partial_{x} \mathcal{H}\left[\left(f_{1}-g_{1}\right)-\left(f_{2}-g_{2}\right)\right]\left(f_{2}-g_{2}\right) .
\end{array}
$$

And we also can do the similar transform for $L_{2}$. Applying the inequality 


$$
\|a+b\|^{2} \leq C_{4}\left(\|a\|^{2}+\|b\|^{2}\right)
$$

again, we have

$$
\begin{array}{r}
\left\|\frac{1}{2} \mathcal{H}\left(f_{1}+g_{1}\right) \partial_{x}\left(f_{1}-g_{1}\right)-\frac{1}{2} \mathcal{H}\left(f_{2}+g_{2}\right) \partial_{x}\left(f_{2}-g_{2}\right)\right\|_{s-2}^{2} \\
\leq C_{7}\left(\left\|\frac{1}{2} \mathcal{H}\left(f_{1}+g_{1}\right) \partial_{x}\left[\left(f_{1}-g_{1}\right)-\left(f_{2}-g_{2}\right)\right]\right\|_{s-2}^{2}\right. \\
\left.\left.\quad-\| \frac{1}{2} \mathcal{H}\left[\left(f_{1}-g_{1}\right)-\left(f_{2}-g_{2}\right)\right]\right) \partial_{x}\left(f_{2}-g_{2}\right) \|_{s-2}^{2}\right) \\
\leq C_{8}\left\|\frac{1}{2} \mathcal{H}\left(f_{1}+g_{1}\right)\right\|_{s-2}^{2}\left\|\partial_{x}\left[\left(f_{1}-g_{1}\right)-\left(f_{2}-g_{2}\right)\right]\right\|_{s-2}^{2} \\
-\left\|\frac{1}{2} \mathcal{H}\left[\left(f_{1}-g_{1}\right)-\left(f_{2}-g_{2}\right)\right]\right\|_{s-2}^{2}\left\|\partial_{x}\left(f_{2}-g_{2}\right)\right\|_{s-2}^{2} .
\end{array}
$$

Note that $f_{1}, g_{1}, f_{2}, g_{2}$ are all in the closed ball, and $\mathcal{H}$ is a bounded operator. Consider the inequality

$$
\left\|\partial_{x} a\right\|_{s} \leq C_{9}\|a\|_{s+1}, \quad\|a\|_{s} \leq C_{10}\|a\|_{s+1},
$$

for arbitrary $a$, we obtain that:

$$
\mathcal{F}\left(\begin{array}{l}
0 \\
0
\end{array}\right)=\left(\begin{array}{l}
0 \\
0
\end{array}\right)
$$

and there exists a constant $C$ for which

$$
\left\|\mathcal{F}\left(\begin{array}{l}
f_{1} \\
g_{1}
\end{array}\right)-\mathcal{F}\left(\begin{array}{l}
f_{2} \\
g_{2}
\end{array}\right)\right\| \leq C R\left\|\left(\begin{array}{l}
f_{1} \\
g_{1}
\end{array}\right)-\left(\begin{array}{l}
f_{2} \\
g_{2}
\end{array}\right)\right\|
$$

whenever $\left(f_{1}, g_{1}\right)$ and $\left(f_{2}, g_{2}\right)$ are selected from the closed ball $\bar{B}_{R}$ of radius $R$ and center at $\mathbf{0}$. If we take some fixed

$$
\left(v_{0}, w_{0}\right) \in H^{s}\left(\mathbb{R}^{2}\right),
$$


then we can prove on the basis that the mapping $(\tilde{v}, \tilde{w}) \mapsto(v, w)$, with

$$
\left(\begin{array}{c}
v \\
w
\end{array}\right)=S(t)\left(\begin{array}{c}
v_{0} \\
w_{0}
\end{array}\right)+\int_{0}^{t} S(t-s) \mathcal{F}\left(\begin{array}{c}
\tilde{v} \\
\tilde{w}
\end{array}\right) d s
$$

is a contraction of $\bar{B}_{R}$ into itself for $R$ sufficiently large [16]. Now we consider the following lemma:

Lemma 3.1 If $\mathcal{H}$ has order 0 , then it is bounded mapping of $L_{p}(\mathbb{R})$ for $1 \leq p \leq \infty$. The operator $\mathcal{H}$ is also a bounded mapping with bounded inverse $H^{s}(\mathbb{R})$ onto itself for $s \geq 0$.

Then we can conclude the following theorem

Theorem 3.2 If $\mathcal{H}$ has order 0, assume that the condition in Eq.(5.36) holds. Let $s \geq 0$ and $(\phi, \psi) \in\left(H^{s}(\mathbb{R})\right)^{2}$. Then there exist $T>0$ and a unique solution pair $(\eta, u)$ in $C\left(0, T ;\left(H^{s}(\mathbb{R})\right)^{2}\right)$ for the system of Eq.(5.32) and Eq.(5.33). Additionally, $\left(\eta_{t}, u_{t}\right) \in C\left(0, T: H^{s-1}(\mathbb{R})\right)^{2}$.Moreover, the correspondence associating initial data to the solution is locally Lipschitz continuous.

The proof for $\mathcal{H}$ of order -1 and 1 of $\mathcal{H}$ are similar. Then we have:

Theorem3.3 If $\mathcal{H}$ has order -1 , let $(\phi, \quad \psi) \in H^{s+1} \times H^{s}, s \geq 0$. Then there exist $T>0$ and a unique solution $(\eta, u)$ in $C\left(0, T: H^{s+1}(\mathbb{R})\right) \times C\left(0, T: H^{s}(\mathbb{R})\right)$ for the system of Eq.(5.32) and Eq.(5.33). Moreover, $\left(\eta_{t}, u_{t}\right) \in C\left(0, T: H^{s+1}(\mathbb{R})\right) \times$ $C\left(0, T: H^{s}(\mathbb{R})\right)$. The correspondence between initial values and solutions is locally Lipschitz.

Theorem 3.3 If $\mathcal{H}$ has order 1 , let $(\phi, \quad \psi) \in H^{s+1} \times H^{s}, s \geq 0$. Then there exist $T>0$ and a unique solution $(\eta, u)$ in $C\left(0, T: H^{s}(\mathbb{R})\right) \times C\left(0, T: H^{s+1}(\mathbb{R})\right)$ for the system of Eq.(5.32) and Eq.(5.33). Moreover, $\left(\eta_{t}, u_{t}\right) \in C\left(0, T: H^{s}(\mathbb{R})\right) \times$ $C\left(0, T: H^{s+1}(\mathbb{R})\right)$. The correspondence between initial values and solutions is locally Lipschitz. 


\subsection{Solitary waves of the general system}

In order to find solitary waves for the general system, we take the radius $r_{0}$ to be constant. Then for Eq.(4.14) and (4.15), we have

$$
\begin{gathered}
\eta_{t}+\frac{1}{2}\left(r_{0}+\eta\right) u_{x}^{\theta}+\eta_{x} u^{\theta}+r_{0}^{3} a u_{x x x}^{\theta}-r_{0}^{2} b \eta_{x x t}=0, \\
u_{t}^{\theta}+\hat{\beta} \eta_{x}+u^{\theta} u_{x}^{\theta}+\hat{\beta} r_{0}^{2} c \eta_{x x x}-r_{0}^{2} d u_{x x t}^{\theta}=0,
\end{gathered}
$$

where $\hat{\beta}=\frac{E h}{\rho r_{0}^{2}}$. Then we take the ansatz:

$$
\eta=\eta(x-v t), \quad u=u(x-v t)
$$

Now we substitue into Eq.(5.49) and Eq.(5.50) and obtain:

$$
\begin{array}{r}
-v \eta^{\prime}+\frac{1}{2} r_{0} u^{\prime}+\frac{1}{2} \eta u^{\prime}+\eta^{\prime} u+r_{0}^{3} a u^{\prime \prime \prime}+v r_{0}^{2} b \eta^{\prime \prime \prime}=0, \\
-v u^{\prime}+\hat{\beta} \eta^{\prime}+u u^{\prime}+\hat{\beta} r_{0}^{2} c \eta^{\prime \prime \prime}+v r_{0}^{2} d u^{\prime \prime \prime}=0,
\end{array}
$$

where the prime symbol denotes the derivative with respect to $s=x-v t$. In order to simplify the Eq.(5.51) we assume:

$$
u=k \eta
$$

Hence the above equations will be

$$
\begin{aligned}
-v \eta^{\prime}+ & \frac{1}{2} r_{0} k \eta^{\prime}+\frac{1}{2} \eta k \eta^{\prime}+\eta^{\prime} k \eta+r_{0}^{3} a k \eta^{\prime \prime \prime}+v r_{0}^{2} b \eta^{\prime \prime \prime}=0, \\
-v k \eta^{\prime}+\hat{\beta} \eta^{\prime}+k \eta k \eta^{\prime}+\hat{\beta} r_{0}^{2} c \eta^{\prime \prime \prime}+v r_{0}^{2} d k \eta^{\prime \prime \prime} & =0 .
\end{aligned}
$$

Then we collect the terms of different order of derivatives, one obtains

$$
\begin{gathered}
\left(\frac{1}{2} r_{0} k-v\right) \eta^{\prime}+\frac{3 k}{2} \eta \eta^{\prime}+\left(a r_{0}^{3} k+b r_{0}^{2} v\right) \eta^{\prime \prime \prime}=0 \\
(\hat{\beta}-v k) \eta^{\prime}+k^{2} \eta \eta^{\prime}+\left(c \hat{\beta} r_{0}^{2}+d v k r_{0}^{2}\right) \eta^{\prime \prime \prime}=0 .
\end{gathered}
$$

In order to get non-trivial solutions, the two equations need to be identical. 
After some computations, we have

$$
v k=3 \hat{\beta}-r_{0} k^{2}, \quad r_{0} k^{2}=\frac{\hat{\beta}(c-2 b+3 d)}{2 a-3 b+3 d} .
$$

For convenience, we take

$$
R=\frac{\hat{\beta}(c-2 b+3 d)}{2 a-3 b+3 d} .
$$

Thereby we obtain that

$$
v k=3 \hat{\beta}-R, \quad k=\sqrt{\frac{R}{r_{0}}}, \quad v=\sqrt{\frac{R}{r_{0}}}(3 \hat{\beta}-R) .
$$

Now the equation has the following form:

$$
\frac{r_{0}}{R}(2 \hat{\beta}-R) \eta^{\prime}-\frac{r_{0}^{3}}{R}[c \hat{\beta}+d(3 \hat{\beta}-R)] \eta^{\prime \prime \prime}=\eta \eta^{\prime}
$$

Taking

$$
\begin{gathered}
\frac{r_{0}}{R}(2 \hat{\beta}-R)=\hat{A}, \\
\frac{r_{0}^{3}}{R}[c \hat{\beta}+d(3 \hat{\beta}-R)]=\hat{B} .
\end{gathered}
$$

Then we know that the equation has solitary wave solution when [7]:

$$
\hat{A} \hat{B}>0
$$

and the solution is

$$
\eta(\xi)=3 \hat{A} \operatorname{sech}^{2}\left(\frac{1}{2} \sqrt{\frac{\hat{A}}{\hat{B}}}\left(\xi+\xi_{0}\right)\right),
$$

where $v_{0}$ is constant. 


\subsection{Existence of solitary waves}

Here we consider the form of $a, b, c$ and $d$.

$$
\begin{gathered}
a=\frac{\left(2 \theta^{2}-1\right) \nu}{16}, \quad b=\frac{\left(2 \theta^{2}-1\right)(1-\nu)}{8}, \\
c=\frac{\left(2 \alpha+\left(1-\theta^{2}\right)\right) \mu}{4}, \quad d=\frac{\left(2 \alpha+\left(1-\theta^{2}\right)\right)(1-\mu)}{4} .
\end{gathered}
$$

Next we study the existence of the solitary waves with the form of Eq.(5.55) in the following systems:

(i) For the classical Boussinesq system, we have

$$
\theta^{2}=\frac{1}{2}, \quad \nu \text { is arbitray, } \mu=0 \text {. }
$$

Then $a, b, c$ are all 0 , and $d>0$. For the system of Eq.(5.53), we can find that the coefficient of $\eta^{\prime \prime \prime}$ in the first equation is 0 , and that the coefficient of $\eta^{\prime \prime \prime}$ in the second equation is $d v k r_{0}^{2}$. Obviously by our definition in order to obtain the non-trivial solution, none of $v, k$ and $r_{0}$ can be 0 . In the condition of classical Boussinesq system, $d$ is not 0 too. So the coefficient of $\eta^{\prime \prime \prime}$ in the second equation can't be 0 too. Then these two equations can't be identical. So we don't have the solitary wave solution of Eq.(5.55).

(ii) For a Bona-Smith type system: $\nu=0, \mu \in \mathbb{R}$, provided $(2 \alpha+(1-$ $\left.\left.\theta^{2}\right)\right) \mu<0, b>0, d>0, a=0, c<0$. Since $c<0,\left(2 \alpha+\left(1-\theta^{2}\right)\right)>0$, we know that $\mu<0$, then we know that $1-\mu>0$, thus $d>0$. To let $b>0$, we have

$$
2 \theta^{2}-1>0
$$

which means that

$$
\theta^{2}>\frac{1}{2}
$$

(iii) For a BBM-BBM type system, we have

$$
\mu=\nu=0 \text {. }
$$

Hence we have that 


$$
b=\frac{\left(2 \theta^{2}-1\right)}{8}, \quad d=\frac{\left(2 \alpha+\left(1-\theta^{2}\right)\right)}{4} .
$$

In order to make the two equations in Eq.(5.53) identical, the following condition must be satisfied:

$$
k^{2} v r_{0}^{2} b=\frac{3}{2} v k^{2} r_{0}^{2} d
$$

which means that

$$
b=\frac{3}{2} d
$$

thus we can obtain the relation for $\theta$ :

$$
2 \theta^{2}-1=3\left(2 \alpha+\left(1-\theta^{2}\right)\right)
$$

Hence we know that the system has the solitary wave solution of form Eq.(5.55) if and only if

$$
\theta^{2}=\frac{6}{5} \alpha+\frac{3}{5}
$$

However, we can see that since $b=\frac{3}{2} d, r_{0} k^{2}=0$. By our definition neither of $r_{0}$ and $k^{2}$ is 0 , so we can't find solitary wave solutions of the form Eq.(5.55).

(iv) For a KdV-KdV system, we have

$$
\nu=1, \quad \mu=1,
$$

Then we know that

$$
a=\frac{\left(2 \theta^{2}-1\right)}{8}, \quad c=\frac{2 \alpha+\left(1-\theta^{2}\right)}{4}
$$

Then we have

$$
R=\frac{c}{2 a} \text {. }
$$

Since $0 \leq \theta^{2} \leq 1$, we have $c>0$. Then we can compute for $\hat{A} \hat{B}>0$, which 
leads to:

$$
\left(2 \hat{\beta}-\hat{\beta} \frac{c}{2 a}\right)(c \hat{\beta})>0 .
$$

Since $\hat{\beta}, c>0$, we have:

$$
\frac{c}{2 a}<2
$$

If $a<0$, the above inequality holds. If $a>0$, then

$$
c<4 a,
$$

thus

$$
2 \alpha+\left(1-\theta^{2}\right)<4 \theta^{2}-2
$$

which leads to:

$$
\theta^{2}>\frac{2 \alpha+3}{5}
$$

(v)For a KdV-BBM system, we have

$$
\nu=0, \quad \mu=1,
$$

Then we know that

$$
\begin{gathered}
d=\frac{\left(2 \alpha+\left(1-\theta^{2}\right)\right)}{4}, \quad a=\frac{2 \theta^{2}-1}{16}, \\
b=c=0 .
\end{gathered}
$$

Then we know that under this condition, we can compute for $\hat{A}, \hat{B}$

$$
\hat{A}=\frac{r_{0}\left(\theta^{2}-6 \alpha-2\right)}{3\left(\theta^{2}-1-2 \alpha\right)}, \quad \hat{B}=\alpha r_{0}^{3}-\frac{1}{4} r_{0}^{3} \theta^{2}+\frac{3}{8} r_{0}^{3} .
$$

In order to satisfy that $\hat{A} \hat{B}>0$, we need:

$$
\frac{6+34 \alpha+48 \alpha^{2}-7 \theta^{2}-20 \alpha \theta^{2}+2 \theta^{4}}{24\left(1+2 \alpha-\theta^{2}\right)}>0 .
$$




\subsection{Symmetries}

To study the symmetry properties of the solution, we computed the symmetry point transformations of the system of Eq.(4.22) and Eq.(4.23) for all $\mu, \nu$ and $\theta$. The solutions remain symmetric with respect to time and space translations. However, if we assume that $\nu=0$ and $\theta^{2}=\frac{1}{2}$, we obtain an additional symmetry transformations. The complete set of infinitesimal generators in this particular case is given here

$$
\begin{gathered}
X_{1}=\frac{\partial}{\partial x}, \\
X_{2}=\frac{\partial}{\partial t}, \\
X_{3}=-t \frac{\partial}{\partial t}+2\left(\eta+r_{0}\right) \frac{\partial}{\partial \eta}+u \frac{\partial}{\partial u} .
\end{gathered}
$$

The corresponding point transformations are given below in the same order:

$$
\begin{gathered}
t^{\prime}=t, \quad x^{\prime}=x, \quad \eta^{\prime}=\eta, \quad u^{\prime}=u, \\
t^{\prime}=t+\epsilon_{2}, \quad x^{\prime}=x, \quad \eta^{\prime}=\eta, \quad u^{\prime}=u, \\
t^{\prime}=e^{-\epsilon_{3}} t, \quad x^{\prime}=x, \quad \eta^{\prime}=-r_{0}+e^{2 \epsilon_{3}}\left(r_{0}+\eta\right), \quad u^{\prime}=-\epsilon_{3} u,
\end{gathered}
$$

where $\epsilon_{1,2,3} \in \mathbb{R}$ are free parameters. The last transformation is a scaling transformation. In order to achieve further symmetries to the solutions one should include higher order terms even if they break the asymptotic order of the models. 


\section{Chapter 6}

\section{Alternative derivation of the Boussinesq system}

In this chapter we provide an alternative derivation of the general system considering the equations of potential flow. We show that the two different derivations lead to the same system. The alternative derivation is based on asymptotic series expansions.

\subsection{Derivation of the additional system}

Now we apply the method of potential flow to solve the problem. Here we consider the dimensional variables first. Since the velocity $\vec{u}$ of the blood is a vector field, we consider a potential $\phi$ such that:

$$
\nabla \phi=\vec{u}
$$

We still denote $u$ the velocity in the $x$ direction and $v$ in the radius direction. Then we have that

$$
\phi_{x}=u, \quad \phi_{r}=v
$$


Hence the Euler equations take the form

$$
\begin{gathered}
\phi_{x x}+\frac{1}{r}\left(r \phi_{r}\right)_{r}=0, \\
\phi_{t}+\frac{1}{2}|\nabla \phi|^{2}+\frac{p}{\rho}=0 .
\end{gathered}
$$

The boundary conditions now have the form

$$
\begin{array}{r}
\phi_{x x}+\frac{1}{r}\left(r \phi_{r}\right)_{r}=0, \quad 0<r \leq r_{0}+\eta, \\
\phi_{r}=\eta_{t}+\left(r_{0 x}+\eta_{x}\right) \phi_{x}, \quad r=r_{0}+\eta, \\
\phi_{t}+\frac{1}{2}|\nabla \phi|^{2}+\frac{\rho^{w}}{\rho} h \eta_{t t}+\frac{E h}{\rho r_{0}^{2}} \eta=0, \quad r=r_{0}+\eta, \\
\phi_{r}=0, \quad r=0 .
\end{array}
$$

Then we perform the same scaling as in the Chapter 3:

$$
\begin{gathered}
\bar{x}=\bar{\Lambda} x, \quad \bar{r}=\bar{R} r, \quad \bar{\eta}=\bar{A} \eta, \quad \bar{t}=\frac{\bar{\Lambda}}{\bar{c}} t, \\
\bar{c}=\sqrt{\frac{\bar{E} h}{2 \bar{R} \rho}}, \quad \epsilon=\frac{\bar{A}}{\bar{R}}, \quad \delta=\frac{\bar{R}}{\bar{\Lambda}} .
\end{gathered}
$$

If we add bar on the dimensional variables and take the non-dimensional variables without bar, we make the following change:

$$
\bar{\phi}=\frac{\bar{\Lambda} \bar{A} \bar{c}}{\bar{R}} \phi
$$

Then we have that

$$
\begin{gathered}
\delta^{2} \phi_{x x}+\frac{1}{r}\left(r \phi_{r}\right)_{r}=0, \quad 0<r \leq r_{0}+\epsilon \eta, \\
\phi_{r}=\delta^{2} \eta_{t}+\delta^{2}\left(r_{0 x}+\epsilon \eta_{x}\right) \phi_{x}, \quad r=r_{0}+\epsilon \eta, \\
\delta^{2} \phi_{t}+\frac{1}{2} \epsilon \delta^{2} \phi_{x}^{2}+\frac{1}{2} \epsilon \phi_{r}^{2}+\delta^{4} \alpha \eta_{t t}+\delta^{2} \beta \eta=0, \quad r=r_{0}+\epsilon \eta,
\end{gathered}
$$




$$
\phi_{r}=0, \quad r=0
$$

Now we take a series expansion with respect to $r$ for the potential function $\phi$ :

$$
\phi=\phi_{0}+r \phi_{1}+r^{2} \phi_{2}+\ldots
$$

Since $\phi$ is only a function of $x$ and $t$, we have

$$
\phi_{r}=\phi_{1}+2 r \phi_{2}+3 r^{2} \phi_{2}+\ldots+n r^{n-1} \phi_{n}+\ldots
$$

and

$$
\frac{1}{r}\left(r \phi_{r}\right)_{r}=\frac{1}{r} \phi_{1}+2^{2} \phi_{2}+3^{3} r \phi_{3}+\ldots+n^{2} r^{n-2} \phi_{n}+\ldots
$$

Substituting Eq.(6.11) into Eq.(6.7), we obtain:

$\delta^{2}\left(\phi_{0}+r \phi_{1}+r^{2} \phi_{2}+\ldots\right)_{x x}+\frac{1}{r} \phi_{1}+2^{2} \phi_{2}+3^{3} r \phi_{3}+\ldots+n^{2} r^{n-2} \phi_{n}+\ldots=0$.

Now we can collect the terms of $r$ of different order. It can be easily seen that the term of $r^{-1}$ is zero, and $\phi_{n+2}$ is some constant multiplying the second order derivative of $\phi_{n}$. Then we observe the following relation:

$$
\delta^{2} \partial_{x}^{2} \phi_{n-2}+n^{2} \phi_{n}=0, \quad n \geq 2
$$

Instantly we find that

$$
\begin{aligned}
\delta^{2} \partial_{x}^{2} \phi_{0}+4 \phi_{2} & =0 \\
\delta^{2} \partial_{x}^{2} \phi_{2}+16 \phi_{4} & =0
\end{aligned}
$$

Since the term of $r^{-1}$ is zero, which is $\phi_{1}$. then by Eq.(6.13) we know that $\phi_{3}, \phi_{5}, \ldots$, and all other $\phi_{n}$ for odd $n$ are all zero:

$$
\phi_{1}=\phi_{3}=\phi_{5}=\ldots=\phi_{2 n-1}=0, \quad n=1,2,3, \ldots
$$


For even $n$ the terms $\phi_{n}$ are:

$$
\phi_{2 n}=\delta^{2 n} \partial_{x}^{2 n} \frac{1}{(-1)^{n}\left(2^{2 n}\right)(n !)^{2}} \phi_{0}
$$

where $n$ are positive integers. Then we can write the $\phi$ in the following form:

$$
\phi=\phi_{0}+r^{2 n} \Sigma_{n=1}^{\infty} \phi_{2 n} \delta^{2 n} \partial_{x}^{2 n} \frac{1}{(-1)^{n}\left(2^{2 n}\right)(n !)^{2}} \phi_{0} .
$$

If only the first several terms are considered, we have:

$$
\phi=\phi_{0}-\frac{r^{2}}{4} \delta^{2} \phi_{0 x x}+\frac{r^{4}}{64} \delta^{4} \phi_{0 x x x x}
$$

Substituting Eq.(6.16) into Eq.(6.8), one obtains

$$
-\frac{2 r}{4} \delta^{2} \phi_{0 x x}+\frac{1}{64} 4 r^{3} \delta^{4} \phi_{0 x x x x}=\delta^{2} \eta_{t}+\delta^{2}\left[r_{0 x}+\epsilon \eta_{x}\right]\left[\phi_{0 x}+r \phi_{1 x}+r^{2} \phi_{2 x} \ldots\right] .
$$

If we assume that only the first a few terms are important, and combine with Eq.(6.11), one obtains that:

$$
\begin{array}{r}
-\frac{1}{2}\left(r_{0}+\epsilon \eta\right) \delta^{2} \phi_{0 x x}+\frac{1}{16}\left(r_{0}+\epsilon \eta\right)^{3} \delta^{4} \phi_{0 x x x x} \\
=\delta^{2} \eta_{t}+\delta^{2}\left[r_{0 x}+\epsilon \eta_{x}\right]\left[\phi_{0 x}-\frac{1}{4}\left(r_{0}+\epsilon \eta\right)^{2} \delta^{2} \phi_{0 x x x}\right] .
\end{array}
$$

After dividing both sides by $\delta^{2}$, one obtains

$$
\begin{array}{r}
-\frac{1}{2}\left(r_{0}+\epsilon \eta\right) \phi_{0 x x}+\frac{1}{16}\left(r_{0}+\epsilon \eta\right)^{3} \delta^{2} \phi_{0 x x x x} \\
=\eta_{t}+\left[r_{0 x}+\epsilon \eta_{x}\right]\left[\phi_{0 x}-\frac{1}{4}\left(r_{0}+\epsilon \eta\right)^{2} \delta^{2} \phi_{0 x x x}\right] .
\end{array}
$$

Thus we have that

$$
\begin{array}{r}
\eta_{t}+\frac{1}{2} r_{0} \phi_{0 x x}+\frac{1}{2} \epsilon \eta \phi_{0 x x}+r_{0 x} \phi_{0 x}+\epsilon \eta_{x} \phi_{0 x} \\
-\frac{\delta^{2}}{4}\left(r_{0}+\epsilon \eta\right)^{2} r_{0 x} \phi_{0 x x x}-\frac{1}{16} r_{0}^{3} \delta^{2} \phi_{0 x x x x}=O\left(\epsilon \delta^{2}\right) .
\end{array}
$$


And we discard the high order terms on the right hand side, one obtains:

$$
\begin{gathered}
\eta_{t}+\frac{1}{2} r_{0} \phi_{0 x x}+\frac{1}{2} \epsilon \eta \phi_{0 x x}+r_{0 x} \phi_{0 x}+\epsilon \eta_{x} \phi_{0 x} \\
-\frac{\delta^{2}}{4}\left(r_{0}+\epsilon \eta\right)^{2} r_{0 x} \phi_{0 x x x}-\frac{1}{16} r_{0}^{3} \delta^{2} \phi_{0 x x x x}=0 .
\end{gathered}
$$

For Eq.(6.9), first we differentiate both sides with respect to $x$, which leads to:

$$
\delta^{2} \phi_{x t}+\frac{\epsilon \delta^{2}}{2} \phi_{x}^{2}+\delta^{4} \alpha \eta_{x t t}+\delta^{2} \beta \eta_{x}=O\left(\epsilon \delta^{2}\right) .
$$

Now we only take the first two terms of Eq.(6.16):

$$
\phi=\phi_{0}-\frac{r^{2}}{4} \delta^{2} \phi_{0 x x}
$$

If we substitute Eq.(6.22) into Eq.(6.21), and take $r=r_{0}$, we will obtain that: $\phi_{0 x t}-\frac{1}{4} \delta^{2} r_{0}^{2} \phi_{0 x x x t}-\frac{r_{0} r_{0 x}}{2} \phi_{0 x x t}+(\beta(x) \eta)_{x}+\epsilon \phi_{0 x} \phi_{0 x x}+\delta^{2} \alpha \eta_{x t t}=O\left(\epsilon, \delta^{2}\right)$.

Since we have $\phi_{0 x}=u_{0}$, which is velocity at the vessel wall, we can see that the above equation is totally the same one as Eq.(3.47). Esssentially the relation for $u_{0}$ and the velocity at $r, u^{r}$, are the same, too. So the system derived here is the same with the one we derived in chapter 3 :

$$
\begin{gathered}
\eta_{t}+r_{0 x} u_{0}+\epsilon \eta_{x} u_{0}+\frac{r_{0}}{2} u_{0} x+\frac{\epsilon \eta}{2} u_{0} x-\delta^{2} u_{0 x x} \frac{\left(r_{0}\right)^{2} r_{0 x}}{4}-\delta^{2} u_{0 x x x} \frac{\left(r_{0}\right)^{3}}{16}=O\left(\epsilon \delta^{2}\right), \\
u_{0 t}+\epsilon u_{0} u_{0 x}+p_{x}^{w}-\delta^{2} u_{0 x x t} \frac{\left(r_{0}\right)^{2}}{4}-\delta^{2} u_{0 x t} \frac{r_{0} r_{0 x}}{2}=0
\end{gathered}
$$

where $p_{x}^{w}=\delta^{2} \alpha \eta_{x t t}-(\beta \eta)_{x}$.

\subsection{The general system}

Here we focus on the vessel with constant radius. We consider a parameter $\theta$, with

$$
0 \leq \theta \leq 1
$$


then we can take that

$$
r=\theta r_{0}
$$

Now we denote the velocity for any parameter $\theta$ by $w$. Obviously when $\theta=0$ we obatin $u_{0}$, which is the horizontal velocity of the center of the vessel. For convenience we only write it as $u$. Since we have done the scaling and we can take $R=r_{0}$, thereby

$$
w=u-\frac{1}{4} \delta^{2} u_{x x}+\frac{1}{64} \delta^{2} u_{x x x x}+O\left(\delta^{6}\right) .
$$

Then we can apply the Fourier transform and denote the variables after Fourier transform with a hat:

$$
\hat{w}=\left(1+\frac{1}{4} \delta^{2} \theta^{2} k^{2}+\frac{1}{64} \delta^{4} \theta^{2} k^{4}\right) \hat{u}+O\left(\delta^{6}\right) .
$$

Since we have the approximation of

$$
\frac{1}{1+x}=1-x+x^{2} \ldots
$$

Let the $x$ above be $\frac{1}{4} \delta^{2} \theta^{2} k^{2}+\frac{1}{64} \delta^{4} \theta^{2} k^{4}$, therefor we obtain the expression of $\hat{u}$ :

$$
\hat{u}=\left(1-\frac{1}{4} \delta^{2} \theta^{2} k^{2}-\frac{1}{64} \delta^{4} \theta^{2} k^{4}+\frac{1}{16} \delta^{4} \theta^{4} k^{4}\right)+O\left(\delta^{6}\right)
$$

thereby

$$
\hat{u}=\left(1-\frac{1}{4} \delta^{2} \theta^{2} k^{2}+\frac{3}{64} \delta^{4} \theta^{2} k^{4}\right)+O\left(\delta^{6}\right) .
$$

Now we can apply the inverse Fourier transform, which gives:

$$
u=w+\frac{1}{4} \delta^{2} \theta^{2} w_{x x}+\frac{3}{64} \delta^{4} \theta^{4} w_{x x x x}+O\left(\delta^{6}\right) .
$$

The above result can be substitued into the previous equations:

$$
\eta_{t}+\frac{1}{2} r_{0} u_{0 x}+\frac{1}{2} \epsilon \eta u_{0 x}+\epsilon \eta_{x} u_{0}-\frac{\delta^{2}}{16} r_{0}^{3} u_{0 x x x}=0
$$




$$
\begin{array}{r}
u_{0 t}-\frac{1}{4} \delta^{2} r_{0}^{2} u_{0 x x t}-\frac{1}{2} \delta^{2} r_{0} r_{0 x} u_{0 x t}+(\beta(x) \eta)_{x}+\epsilon u_{0} u_{0 x} \\
-\delta^{2} \alpha\left(\frac{1}{2} r_{0} u_{0 x}\right)_{x t}=0
\end{array}
$$

which leads to:

$$
\begin{gathered}
\eta_{t}+\frac{1}{2}\left(w_{x}+\frac{1}{4} \delta^{2} \theta^{2} w_{x x x}\right)+\frac{1}{2} \epsilon \eta w_{x}+\epsilon \eta_{x} w-\frac{\delta^{2}}{16} w_{x x x}=O\left(\epsilon, \delta^{2}\right) \\
w_{t}-\frac{1}{4} \delta^{2} w_{x x t}+(\beta(x) \eta)_{x}+\epsilon w w_{x}-\delta^{2} \alpha \frac{1}{2} w_{x x t}=O\left(\epsilon, \delta^{2}\right) .
\end{gathered}
$$

\subsection{Viscoelastic correction}

For $\mathrm{Eq}(2.27)$ in chapter 2, we can add one correction term of viscoelastic, then the equation is:

$$
\rho^{w} h \bar{\eta}_{\overline{t t}}=\bar{p}^{w}+\tilde{\gamma} \bar{\eta}_{\bar{t}}-\frac{\bar{E}_{\sigma}(\bar{x}) h}{\bar{r}_{0}^{2}} \bar{\eta}
$$

where $\gamma$ is a constant related to visco-elasticity. In the previous chapters, we have the following equation

$$
\delta^{2} \phi_{t}+\frac{1}{2} \epsilon \delta^{2} \phi_{x}^{2}+\frac{2}{2} \epsilon \phi_{r}^{2}+\delta^{4} \alpha \eta_{t t}+\delta^{2} \beta \eta=0, \quad r=r_{0}+\epsilon \eta .
$$

Since now we import the viscoelastic terms, we have the new equation:

$$
\delta^{2} \phi_{t}+\frac{1}{2} \epsilon \delta^{2} \phi_{x}^{2}+\frac{2}{2} \epsilon \phi_{r}^{2}+\delta^{4} \alpha \eta_{t t}-\delta^{4} \frac{\tilde{\gamma} \bar{R}}{\bar{\Lambda} \bar{A} \bar{c}} \eta_{x t}+\delta^{2} \beta \eta=0, \quad r=r_{0}+\epsilon \eta .
$$

Then we do the similar derivation as in section 6.1, which leads to:

$$
u_{0 t}+\epsilon u_{0} u_{0 x}+\delta^{2} \alpha \eta_{x t t}+(\beta \eta)_{x}-\delta^{2} \frac{\tilde{\gamma} \bar{R}}{\bar{\Lambda} \bar{A} \bar{c}} \eta_{x t}-\delta^{2} u_{0 x x t} \frac{\left(r_{0}\right)^{2}}{4}-\delta^{2} u_{0 x t} \frac{r_{0} r_{0 x}}{2}=0
$$


By substituting Eq.(6.30) into the system of Eq.(4.14) and Eq.(4.15), one obtains the new system:

$$
\begin{gathered}
\eta_{t}+\frac{1}{2}\left(r_{0}+\eta\right) u_{x}^{\theta}+\left(r_{0 x}+\eta_{x}\right) u^{\theta}=0, \\
\left(1-\bar{\alpha} r_{0 x x t}\right) u_{t}^{\theta}+[\beta \overline{(x)} \eta]_{x}+u^{\theta} u_{x}^{\theta}-\frac{\left(3 \alpha+r_{0}\right) r_{0 x}}{2} u_{x t}^{\theta}-\frac{\tilde{\gamma}}{\rho} \eta_{x t} \\
-\frac{\left(4 \bar{\alpha}+r_{0}\right) r_{0}}{8} u_{x x t}^{\theta}+8 \kappa \frac{u^{w}}{r_{0}^{2}}=0 .
\end{gathered}
$$

By Eq.(6.25), one obatins:

$$
u_{t}+(\beta(x) \eta)_{x}=O\left(\epsilon, \delta^{2}\right)
$$

And we also have Eq.(6.24), which lead the relation:

$$
\eta_{t}=-\frac{1}{2} r_{0} u_{0 x}-r_{0 x} u_{0}+O\left(\epsilon, \delta^{2}\right)
$$

Then we can make substitution for the term of $u_{x t}$ and $\eta_{x t}$ of Eq.(6.33), which lead to the following result after going back to the dimensional form:

$$
\begin{gathered}
\eta_{t}+\frac{1}{2}\left(r_{0}+\eta\right) u_{x}^{\theta}+\left(r_{0 x}+\eta_{x}\right) u^{\theta}=0 \\
\left.\left(1-\bar{\alpha} r_{0 x x t}\right) u_{t}^{\theta}+[\beta \overline{(x}) \eta\right]_{x}+u^{\theta} u_{x}^{\theta}-\frac{\left(3 \alpha+r_{0}\right) r_{0 x}}{2}(\beta \overline{(x)} \eta)_{x x}+\frac{\tilde{\gamma}}{\rho}\left(\frac{1}{2} r_{0} u_{0 x}+r_{0 x} u_{0}\right) \\
-\frac{\left(4 \bar{\alpha}+r_{0}\right) r_{0}}{8} u_{x x t}^{\theta}+8 \kappa \frac{u^{w}}{r_{0}^{2}}=0 .
\end{gathered}
$$




\section{Chapter 7}

\section{Numerical Methods}

In this Chapter we introduce the basic concepts of finite difference methods for the numerical solutions of partial differential equations. We develop finite difference methods for the numerical solution of the BBM equation and for the classical Boussinesq system studied in the previous chapters. Finally, we demonstrate the application of the new models to blood flow problems. By performing several numerical simulations.

\subsection{Finite difference method}

Now we consider the funcion $u(x, t)$, which is defined on the domain $x \in[a, b], \times t \in[0, T]$. We define our grids $x_{0}, x_{1}, \ldots, x_{N+1}$ for $x$ and $t_{0}, t_{1}, \ldots, t_{M+1}$ for $t$. Let $\Delta x=x_{i+1}-x_{i}, \Delta t=t_{j+1}-t_{j}$ for any $i, j$ and $\Delta x$ and $\Delta t$ are all uniform. Let $x_{0}=a$ and $x_{N+1}=b$. Let $t_{0}=0$ and $t_{M+1}=T$. Then we denote $u_{i}^{j}$ the approximation of $u\left(x_{i}, t_{j}\right)$ by only taking the $u(x, t)$ at $x=x_{i}$ and $t=t_{j}$ for the $i, j$ invovled. Now we have discretized $u(x, t)$ at the domain $x \in[a, b], t \in[0, T]$.

Thereby we can discretize the derivatives. By the Taylor expansion, we have:

$$
\left.\frac{\partial u}{\partial t}\right|_{i} ^{j}=\frac{u_{i}^{j+1}-u_{i}^{j}}{\Delta t}+O(\Delta t) .
$$

Then we obtain the derivatives with respect to $t$ of the finite difference form 
of order $\Delta t$. By the Taylor expansion also gives that

$$
\begin{aligned}
& u(x+\Delta x, t)=u(x, t)+\frac{\partial u(x, t)}{\partial x} \Delta x+\frac{1}{2} \frac{\partial^{2} u(x, t)}{\partial x^{2}} \Delta x^{2}+O\left(\Delta x^{3}\right), \\
& u(x-\Delta x, t)=u(x, t)-\frac{\partial u(x, t)}{\partial x} \Delta x+\frac{1}{2} \frac{\partial^{2} u(x, t)}{\partial x^{2}} \Delta x^{2}+O\left(\Delta x^{3}\right) .
\end{aligned}
$$

By comparing the above two equations, we can eliminate the terms of order $\Delta x^{2}$, thus we obtain

$$
\left.\frac{\partial u}{\partial x}\right|_{i} ^{j}=\frac{u_{i+1}^{j}-u_{i-1}^{j}}{2 \Delta x}+O\left(\Delta x^{2}\right),
$$

which is the derivative with respect to $x$ of the finite difference form of order $\Delta x^{2}$. By applying the Taylor expansion, we can have the following relation:

$$
\begin{aligned}
& u(x+2 \Delta x, t)=u(x, t)+2 \frac{\partial u(x, t)}{\partial x} \Delta x+2 \frac{\partial^{2} u(x, t)}{\partial x^{2}} \Delta x^{2}+\frac{4}{3} \frac{\partial^{3} u(x, t)}{\partial x^{3}} \Delta x^{3}+O\left(\Delta x^{4}\right), \\
& u(x-2 \Delta x, t)=u(x, t)-2 \frac{\partial u(x, t)}{\partial x} \Delta x+2 \frac{\partial^{2} u(x, t)}{\partial x^{2}} \Delta x^{2}-\frac{4}{3} \frac{\partial^{3} u(x, t)}{\partial x^{3}} \Delta x^{3}+O\left(\Delta x^{4}\right),
\end{aligned}
$$

Substituting Eq.(7.1) and Eq.(7.2) into and Eq.(7.3) and and Eq.(7.4), we obtain the second order derivatives with respect to $x$ of order $\Delta x^{2}$ :

$$
\left.\frac{\partial^{2} u(x, t)}{\partial x^{2}}\right|_{i} ^{j}=\frac{u_{i-1}^{j}-2 u_{i}^{j}+u_{i+1}^{j}}{\Delta x^{2}} .
$$

We will use these formules to descritize our model equation.

\subsection{A finite difference method for the BBM equation}

In this section we study the numerical solution of the BBM equation. The numerical scheme is linearly implict and it was first derived in [18]. For convenience, we take the all the coefficients to be 1 . Then the BBM 
equation has the following form:

$$
u_{t}+u_{x}+u u_{x}+u_{x x t}=0,
$$

in $x \in[a, b]$ with initial condition $u(x, 0)=u_{0}(x)$ and boudary condition $u(a, t)=u(b, t)=0$. We apply the following method [9]:

$$
\Delta_{t}\left(1-\delta_{x}^{2}\right) u_{i}^{j}+\frac{1}{2}\left(1+u_{i}^{j}\right) H_{x}\left(u_{i}^{j+1}+u_{i}^{j}\right)=0 .
$$

The operators here are:

$$
\begin{gathered}
\delta_{x}^{2} u_{i}^{j}=\frac{u_{i-1}^{j}-2 u_{i}^{j}+u_{i+1}^{j}}{\Delta x^{2}}, \\
H_{x} u_{i}^{j}=\frac{u_{i+1}^{j}-u_{i-1}^{j}}{2 \Delta x}, \\
\Delta_{t} u_{i}^{j}=\frac{u_{i}^{j+1}-u_{i}^{j}}{\Delta t} .
\end{gathered}
$$

For convenience. we denote $\Delta x$ by $h$ and denote $\Delta t$ by $\tau$. Then we have the exact form the the finite difference relation:

$$
\begin{array}{r}
\frac{u_{i}^{j+1}-u_{i}^{j}}{\tau}-\frac{\left(u_{i+1}^{j+1}-2 u_{i}^{j+1}+u_{i-1}^{j+1}\right)-\left(u_{i+1}^{j}-2 u_{i}^{j}+u_{i-1}^{j}\right)}{h^{2} \tau} \\
+\frac{1}{2}\left(1+u_{i}^{j}\right) \frac{u_{i+1}^{j+1}-u_{i-1}^{j+1}+u_{i+1}^{j}-u_{i-1}^{j}}{2 h}=0 .
\end{array}
$$

Rearranging the terms we have:

$$
\begin{aligned}
& \frac{u_{i}^{j+1}}{\tau}-\frac{u_{i+1}^{j+1}-2 u_{i}^{j+1}+u_{i-1}^{j+1}}{h^{2} \tau}+\frac{1}{2}\left(1+u_{i}^{j}\right) \frac{u_{i+1}^{j+1}-u_{i-1}^{j+1}}{2 h} \\
& =\frac{u_{i}^{j}}{\tau}-\frac{u_{i+1}^{j}-2 u_{i}^{j}+u_{i-1}^{j}}{h^{2} \tau}-\frac{1}{2}\left(1+u_{i}^{j}\right) \frac{u_{i+1}^{j}-u_{i-1}^{j}}{2 h} .
\end{aligned}
$$

Collecting the $j+1$ terms on the left side of Eq.(7.6) we can express this 
relation in matrix-vector form $C U=F$, where

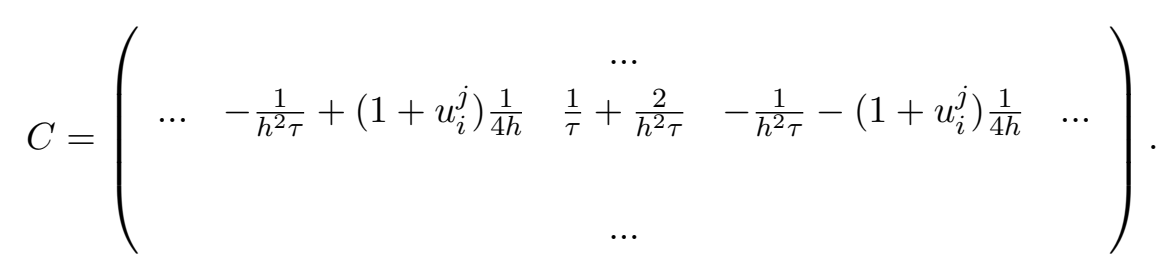

$C$ is tridiagonal with diagonals:

$$
\begin{gathered}
C_{i-1, i}=-\frac{1}{h^{2} \tau}-\left(1+u_{i}^{j}\right) \frac{1}{2 h}, \\
C_{i, i}=\frac{1}{\tau}+\frac{2}{h^{2} \tau}, \\
C_{i+1, i}=\frac{1}{\tau}+\frac{2}{h^{2} \tau} .
\end{gathered}
$$

The first row and the $(N+1)$ th row are related to boundary conditions. And we also consider the vector $U$ for some $j$ :

$$
U^{T}=\left(\begin{array}{lll}
u_{1}^{j+1} & u_{2}^{j+1} \ldots & u_{N+1}^{j+1}
\end{array}\right)
$$

For $F^{T}:=\left(\begin{array}{lll}F_{1} & F_{2} \ldots & F_{N+1}\end{array}\right)$, we have

$$
F_{i}=\frac{u_{i}^{j}}{\tau}-\frac{u_{i+1}^{j}-2 u_{i}^{j}+u_{i-1}^{j}}{h^{2} \tau}-\frac{1}{2}\left(1+u_{i}^{j}\right) \frac{u_{i+1}^{j}-u_{i-1}^{j}}{2 h},
$$

for $2<i<N$. When $i=1$ or $i=N+1$, we take the boundary conditions into account.

The equation $C U=F$ is for some specific $j$. On the left hand side the terms are all related to $j+1$ and on the right hand side the terms are all related to $j$. To solve the system, we multiply $C^{-1}$ on both sides:

$$
U=C^{-1} F
$$

Once all the $u_{i}^{j}$ are known for some $j$ and for all $i$, we can compute the solution for $j+1$. Note that the matrix $C$ depends on $j$ since it has terms of that contain $u_{i}^{j}$. 


\subsection{Stability analysis of the numerical method for the BBM equation}

In this section we study the stability of the numerical scheme. Specifically we study the limits for the discretization parameters $\Delta t, \Delta_{x}$ so that the numerical solutions remains bounded. In order to do so we consider the Von Neumann analysis of the linearized equation.

$$
u_{t}+u_{x}-u_{x x t}=0
$$

Since we will denote the imaginary unit by $i$ here, we will denote $u$ by $u_{n}^{j}$. Then we consider:

$$
u_{n}^{j}=\xi^{j} e^{i k n h}
$$

It can be easily seen that

$$
\begin{gathered}
u_{n}^{j+1}=\xi^{j+1} e^{i k n h} \\
u_{n+1}^{j}=\xi^{j} e^{i k h} e^{i k n h} .
\end{gathered}
$$

Thus for the linearised Eq.(7.5), we have

$$
\frac{(\xi-1)}{\tau}+\frac{(\xi-1)\left(e^{i k h}-2+e^{-i k h}\right)}{h^{2} \tau}+\frac{1}{2}(\xi+1) \frac{e^{i k h}-e^{-i k h}}{2 h}=0 .
$$

Since we have

$$
\begin{gathered}
e^{i k h}+e^{-i k h}=2 \cos (k h), \\
e^{i k h}-e^{-i k h}=2 \sin (k h),
\end{gathered}
$$

we obtain that

$$
\frac{(\xi-1)}{\tau}+\frac{(\xi-1)(2 \cos (k h)-2)}{h^{2} \tau}+\frac{1}{2}(\xi+1) \frac{2 \sin (k h)}{2 h}=0 .
$$

Therefore solving for $\xi$ we have

$$
\xi=\frac{1-\frac{2 \cos (k h)-2}{h^{2}}+i \frac{\sin (k h)}{2 h}}{1-\frac{2 \cos (k h)-2}{h^{2}}-i \frac{\sin (k h)}{2 h}} .
$$


In order for the solutions to be bounded, so we have $|\xi| \leq 1$. Observe that the denominator and the numerator of $\xi$ are complex conjugate, we have that

$$
|\xi|=1
$$

for all $k$ and $h$. So we conclude the solution is bounded for any value $k, \Delta t$, $\Delta x$ and this numerical scheme is unconditionally stable.

\subsection{A numerical method for the Boussinesq system}

In this section we develop a finite difference method to solve the Boussinesq system Eq.(4.58) and Eq.(4.59):

$$
\begin{gathered}
\eta_{t}+\frac{1}{2}\left(r_{0}+\eta\right) u_{x}^{\theta}+\left(r_{0 x}+\eta_{x}\right) u^{\theta}=0, \\
\left(1-\bar{\alpha} r_{0 x x}\right) u_{t}^{\theta}+[\overline{(x)} \eta]_{x}+u^{\theta} u_{x}^{\theta}-\frac{\left(3 \alpha+r_{0}\right) r_{0 x}}{2} u_{x t}^{\theta} \\
-\frac{\left(4 \bar{\alpha}+r_{0}\right) r_{0}}{8} u_{x x t}^{\theta}+8 \kappa \frac{u^{w}}{r_{0}^{2}}=0 .
\end{gathered}
$$

We consider similar approximations as in the previous sections to derive the method of [18]. We discretise the time interval by $\tau$ and spatial interval by $h$. We denote $U_{i}^{j} \approx u\left(x_{i}, t_{j}\right)$ and $H_{i}^{j} \approx \eta\left(x_{i}, t_{j}\right)$ instead of eta. From Eq.(7.7) we obtain that:

$$
\frac{H_{i}^{j+1}-H_{i}^{j}}{\tau}+\frac{1}{2}\left(r_{0 i}+H_{i}^{j}\right) \frac{U_{i+1}^{j}-U_{i-1}^{j}}{2 h}+\left(r_{0 x i}+\frac{H_{i+1}^{j}-H_{i-1}^{j}}{2 h}\right) U_{i}^{j}=0,
$$

By the above equation, we can solve for $H_{i}^{j+1}$. Since we have the term of $j+1$, we can take the average term:

$$
\frac{H_{i}^{j}+H_{i}^{j+1}}{2}
$$

instead of the term $H_{i}^{j}$. For covenience, we denote the average term by 
$H_{i}^{j+\frac{1}{2}}$. We also take that:

$$
B H_{i}^{j}=\bar{\beta}_{i} H_{i}^{j} .
$$

Therefore we can compute the $U_{i}^{j+1}$ by Eq.(7.8) using the following relation:

$$
\begin{array}{r}
P(i) \frac{U_{i}^{j+1}-U_{i}^{j}}{\tau}+\frac{B H_{i+1}^{j+\frac{1}{2}}-B H_{i-1}^{j+\frac{1}{2}}}{2 h} \\
+\frac{U_{i+1}^{j}-U_{i-1}^{j} U_{i}^{j}+Q(i) \frac{B H_{i+1}^{j+\frac{1}{2}}-2 B H_{i}^{j+\frac{1}{2}}+B H_{i-1}^{j+\frac{1}{2}}}{2 h}}{h^{2}} \\
-R(i) \frac{\left(U_{i+1}^{j+1}-2 U_{i}^{j+1}+U_{i-1}^{j+1}\right)-\left(U_{i+1}^{j}-2 U_{i}^{j}+U_{i-1}^{j}\right)}{h^{2} \tau} \\
+8 \kappa \frac{U_{i}^{j}}{r_{0 i}^{2}}=0 .
\end{array}
$$

Now we can solve for $U_{i}^{j+1}$ and the process is similar to the previous section. Finally at every time step we improve the first approximation of $H_{i}^{j}$ obtained by Eq.(7.9) using the average term of $U_{i}^{j+\frac{1}{2}}$ :

$$
\frac{H_{i}^{j+1}-H_{i}^{j}}{\tau}+\frac{1}{2}\left(r_{0 i}+H_{i}^{j}\right) \frac{U_{i+1}^{j+\frac{1}{2}}+-U_{i-1}^{j+\frac{1}{2}}}{2 h}+\left(r_{0 x i}+\frac{H_{i+1}^{j}-H_{i-1}^{j}}{2 h}\right) U_{i}^{j}=0 .
$$

\subsection{Numerical experiments}

In order to validate our system, we perform some numerical experiments. Table 7.1 presents the parameters used in our experiments, which is from the reference [23]. We consider a vessel with the parameters of table 7.1 and we study the propagation of a pulse in an elastoc vessel.

Now we compute the relations for a stenosed vessel, tapered vessel and a vessel with constant radius. The parameter of the studied vessel is listed in the above table. We let the inflow side of the vessel to be $x=0$ and the outfow side of the vessel to be $x=0.126$. 


\begin{tabular}{ccc}
\hline Parameter & corresponding letter & \\
\hline Vessel's length & $L$ & $1.26 \times 10^{-2} \mathrm{~m}$ \\
Vessel's nominal radius & $R$ & $3 \times 10^{-3} \mathrm{~m}$ \\
Vessel's thickness & $h$ & $3 \times 10^{-4} \mathrm{~m}$ \\
Wall density & $\rho^{w}$ & $1000 \mathrm{~kg} / \mathrm{m}^{3}$ \\
Blood density & $\rho$ & $1060 \mathrm{~kg} / \mathrm{m}^{3}$ \\
Young's modulus & $E$ & $4.07 \times 10^{5} \mathrm{~kg} /\left(\mathrm{msec}^{2}\right)$ \\
Kinetic viscosity & $\kappa$ & $4 \times 10^{-6} \mathrm{~m} / \mathrm{sec}$ \\
\hline
\end{tabular}

Table 7.1: Parameters in the numerical experiments

To check our system, a stenosed part is involved in the vessel. In this case, the radius can be described by the function:

$$
r_{0}(x)=0.003-0.0003(1-\cos (2 \pi(x-0.02))) / 2, \quad x \in[0.02,0.03] .
$$

We considera tapered vessel also. For the tapered vessel, the radius will reduces linears with respect to $x$. At $x=0.126$, the radius is 0.002 , compared with 0.003 at $x=0$.

We compute the flow rate $q$ and the pressure $p$ in the following figures. The blue lines represent the boundary conditions at $x=0$ while the red lines represent the solution at $x=0.126$.

In the Figure 7.1,we demonstrate the flow rate and the pressure at the position of $x=0$ anb $x=0.126$. In the Figure 7.2 shows that it takes longer time for the wave peak to arrive. Since that the vessel narrows, the velocity can be even larger than the initial velocity. The biggest difference is in the pressure, due to the narrowing of the vessel. In the Figure 7.3, we can see that the flow rate of the blood is a little smaller than the flow rate of the blood in the constant radius vessel.

We can also compare the results obtained with the BBM equation. Since the BBM equation is for inviscid fluid, we take the viscosity 0 for the system as well. The results here shows that the BBM equation is a good aprroximation for the system.

Notice that the pressure here is not the "real" pressure because we also need to consider the pressure ourside the vessel. Here we take the pressure ourside to be 0 just for convenience since the pressure outside is seen to be 

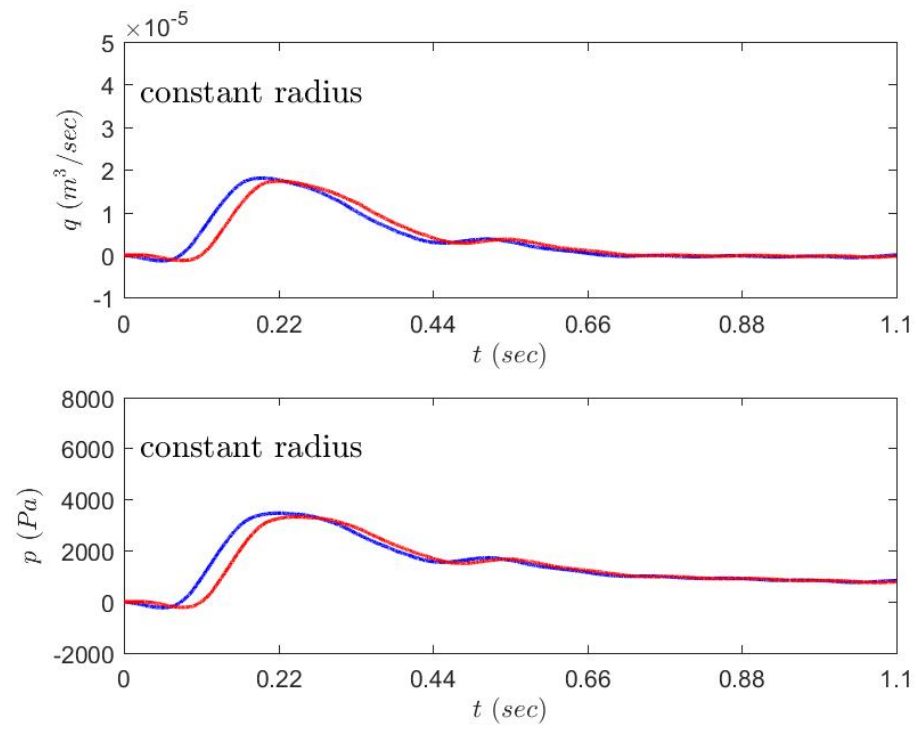

Figure 7.1: vessel with constant raidus

constant and to be equal for all vessels. These results agree with observations in analogous vessels simulating the carotid artery showing that these models can be used to simulate blood flow in arteries. 

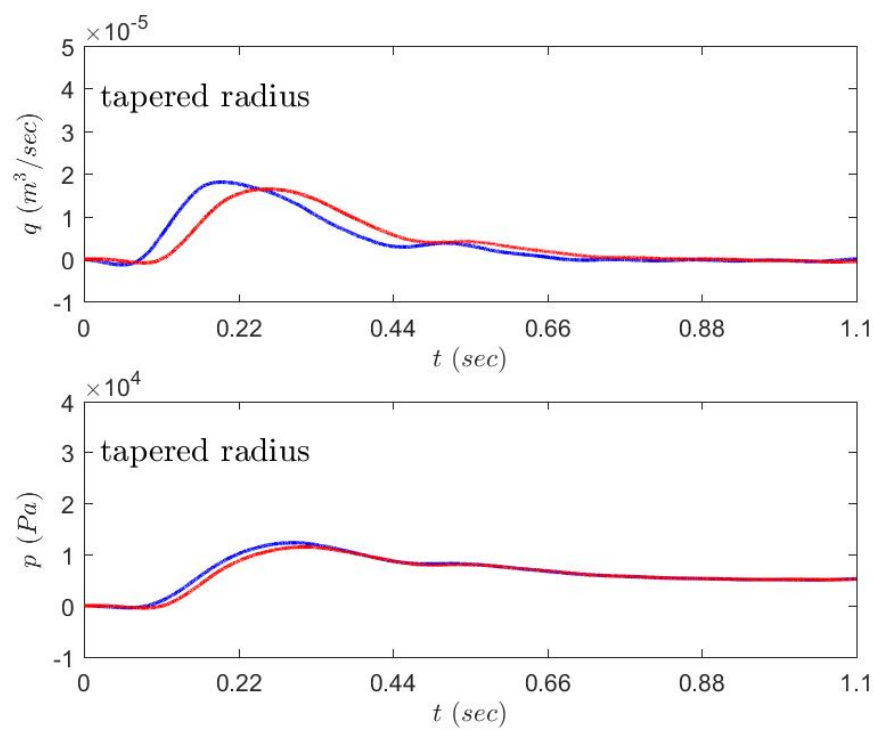

Figure 7.2: tapered vessel
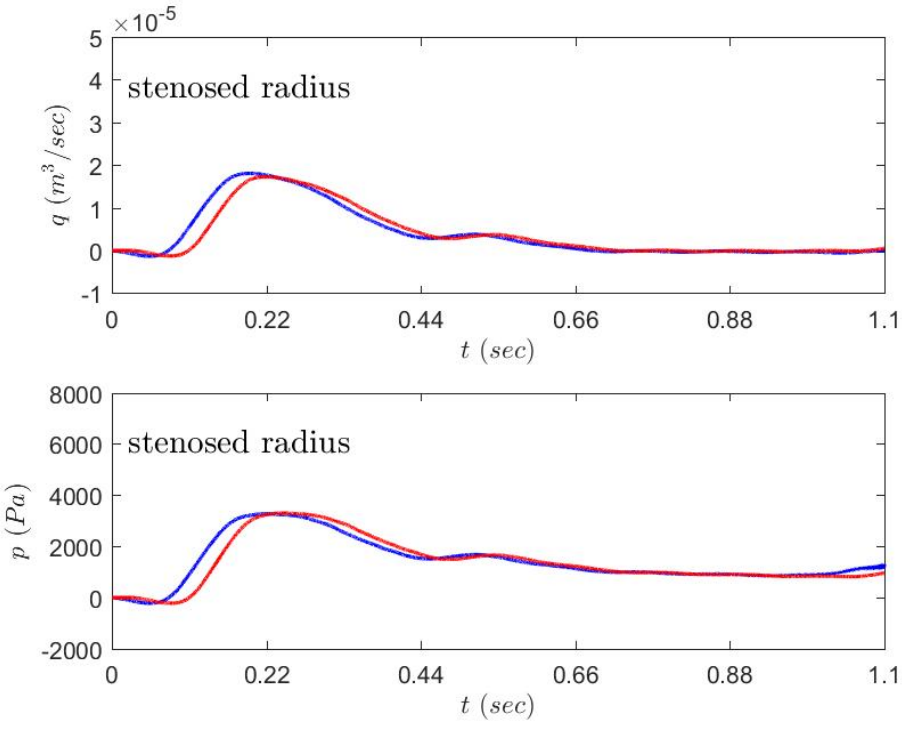

Figure 7.3: stenosed vessel 

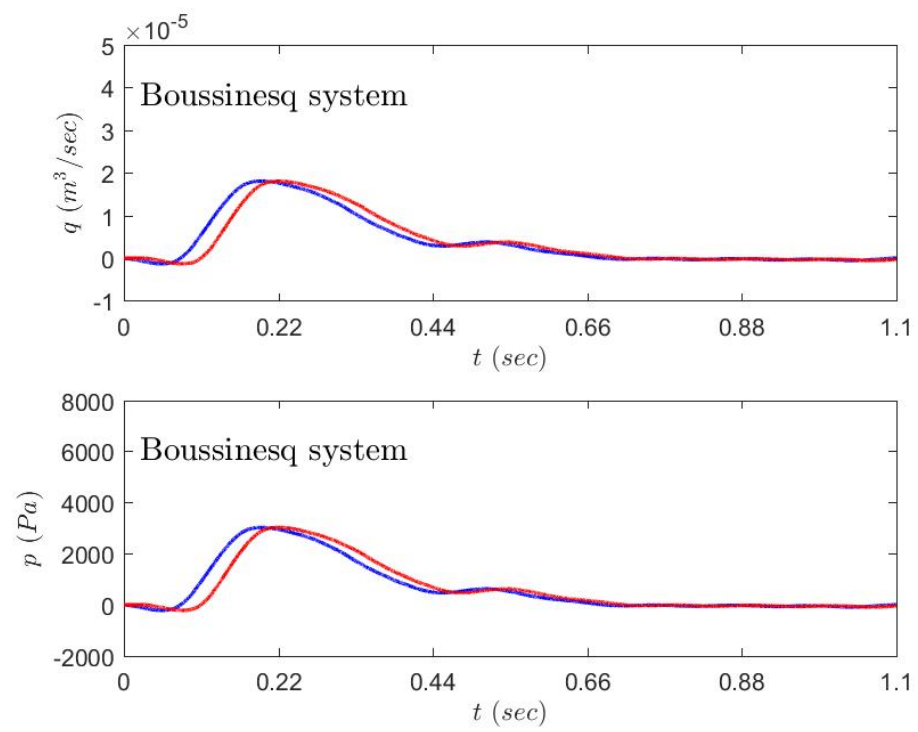

Figure 7.4: system of constant raidus and no viscosity
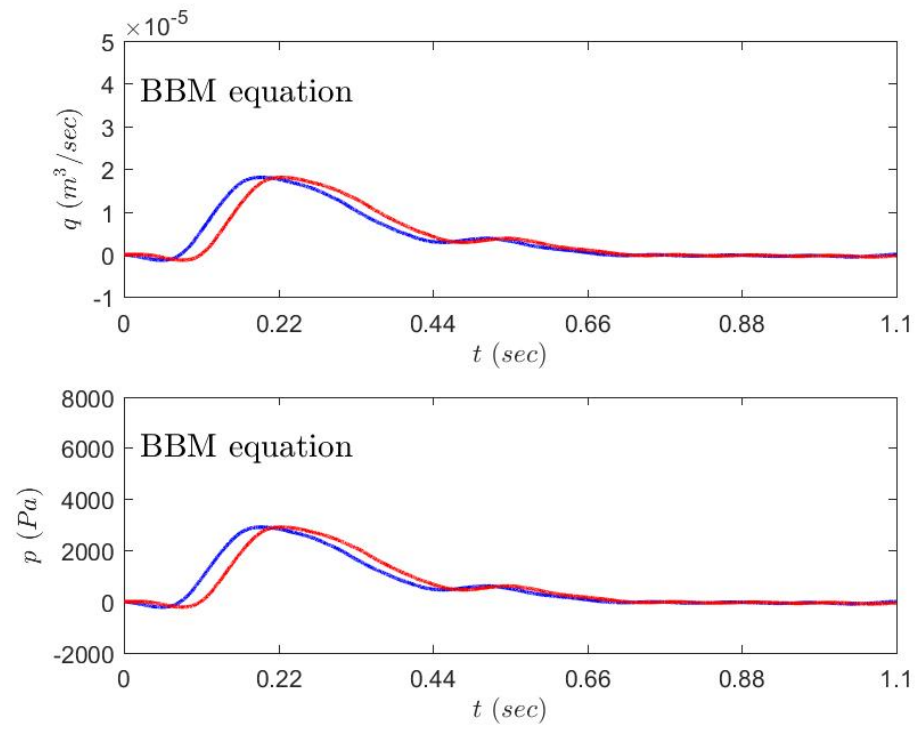

Figure 7.5: BBM equation for the vessel 


\section{Chapter 8}

\section{Conclusions}

In this thesis we review previous derived systems for fluids in elastic vessels with constant radius. We also review the derivation of the equation for the vessel wall. By the no-slip condition on the vessel wall and equation of the vessel wall we derived a Boussinesq system for fluid flow in elastic vessels with variable radius. The velocity we use in the system is that on the vessel wall. Based on this system, we compute soliton solutions and dispersion relations. We also compute the velocity at the center of the vessel.

Then we derive the general system with parameters, and for the general system we can use the velocity at any distance from the center of the vessel. We studied the general system with constant radius in detail. Therefore we can derived one-way propagation models, namely BBM and KdV equations. By some deriviation we add the term of viscotiy thus obtain the dissipative Boussinesq systems.

We compute the dispersion relation of the Euler system, and we compared the dispersion relation of the Euler system and that of the general system. Specifically we distinguish a simple system by adjusting some parameters. It shows that when the phase velocity is not very large, our system is a good approximation of the Euler system. We proved the wellposedness of the linearized system, and for the non-linear system. The existence of the solitary waves has been studied too. Also, some symmetry properties are also involved in the study. 
The method of potential flow and asymptotic series is used as an alternative method to derive the same Boussinesq systems. It shows that the pervious derivation is proper. Application of the Fourier analysis leads to different type of systems.

For the numerical discretization of the systems We introduce the finite difference method in this theis. We discritize numerically the BBM equation and the classical Boussinesq system. We studied the stability of the numerical method for the BBM equation. Some modifications have been imposed in the scheme for the Boussinesq system to improve the results. Several numerical experiments was carried out. The results show that the tapered vessel make the peak exist longer, and the stenosed vessel makes the peak move slower. We also can see that the BBM equation derived as one-way propagation model has good approximation properties for pulse wave in elastic vessels.

In this study, we derived asymptotic models for fluid in elastic vessels, which hasn't been done before. Since we derived a Boussinesq system, we expand the application range of the Boussinesq system. It will be helpful to have more profound understanding of fluids in elastic vessels. The result of this study will help studies in medicine and engineering. 


\section{Bibliography}

[1] ANliker, M., RockWell, R. L., AND OgDen, E. Nonlinear analysis of flow pulses and shock waves in arteries. Zeitschrift für angewandte Mathematik und Physik ZAMP 22, 3 (1971), 563-581.

[2] BocKWINKEL, H. Over de voortplanting van licht in een twee-assig kristal rondom een middelpunt van trilling. Akad. van Wet. Amsterdam Versl. Wis-Natuurk. Afd 14 (1906), 636-651.

[3] BONA, J. L., CHEN, M., AND SAUT, J. Boussinesq equations and other systems for small-amplitude long waves in nonlinear dispersive media: Ii. the nonlinear theory. Nonlinearity 17, 3 (2004), 925.

[4] Bona, J. L., Chen, M., And SAUt, J.-C. Boussinesq equations and other systems for small-amplitude long waves in nonlinear dispersive media. i: Derivation and linear theory. Journal of Nonlinear Science 12, 4 (2002).

[5] CAScaval, R. C. Variable coefficient kdv equations and waves in elastic tubes. LECTURE NOTES IN PURE AND APPLIED MATHEMATICS (2003), 57-70.

[6] CASCAVAL, R. C. A boussinesq model for pressure and flow velocity waves in arterial segments. Mathematics and Computers in Simulation 82, 6 (2012), 1047-1055.

[7] CHEN, M. Exact traveling-wave solutions to bidirectional wave equations. International Journal of Theoretical Physics 37, 5 (1998), 1547-1567. 
[8] DUtYKH, D., AND DiAs, F. Dissipative boussinesq equations. Comptes Rendus Mecanique 335, 9-10 (2007), 559-583.

[9] EILBECK, J., AND MCGuire, G. Numerical study of the regularized long-wave equation i: Numerical methods. Journal of Computational Physics 19, 1 (1975), 43-57.

[10] Euler, L. Principia pro motu sanguinis per arterias determinando. Opera posthuma mathematica et physica anno (1844), 814-823.

[11] Formaggia, L., LAmponi, D., And Quarteroni, A. Onedimensional models for blood flow in arteries. Journal of engineering mathematics 47, 3-4 (2003), 251-276.

[12] FUNG, Y.-C. Biomechanics: circulation. Shock 9, 2 (1998), 155.

[13] FUNG, Y.-C. Biomechanics: circulation. Springer Science \& Business Media, 2013.

[14] Hughes, T. J., And Lubliner, J. On the one-dimensional theory of blood flow in the larger vessels. Mathematical Biosciences 18, 1-2 (1973), 161-170.

[15] KORTEWEG, D. Ueber die fortpflanzungsgeschwindigkeit des schalles in elastischen röhren. Annalen der Physik 241, 12 (1878), 525-542.

[16] KREYSZIG, E. Introductory functional analysis with applications, vol. 1. wiley New York, 1989.

[17] LANDAU, L. D., AND SYKES, J. Fluid mechanics: Vol 6.

[18] Peregrine, D. H. Long waves on a beach. Journal of fluid mechanics 27, 4 (1967), 815-827.

[19] Sherwin, S., Franke, V., Peiró, J., And Parker, K. Onedimensional modelling of a vascular network in space-time variables. Journal of Engineering Mathematics 47, 3 (2003), 217-250. 
[20] Van de Vosse, F. N., And Stergiopulos, N. Pulse wave propagation in the arterial tree. Annual Review of Fluid Mechanics 43 (2011), 467-499.

[21] WitziG, K. Uber erzwungene Wellenbewegungen zaher, inkompressibler Flussigkeiten in elastischen Rohren. PhD thesis, Universitat Bern, 1914.

[22] Womersley, J. R. An elastic tube theory of pulse transmission and oscillatory flow in mammalian arteries. Tech. rep., AEROSPACE RESEARCH LABS WRIGHT-PATTERSON AFB OH, 1957.

[23] Xiao, N., Alastruey, J., And Alberto Figueroa, C. A systematic comparison between 1-d and 3-d hemodynamics in compliant arterial models. International journal for numerical methods in biomedical engineering 30, 2 (2014), 204-231.

[24] YOUnG, T. Hydraulic investigations, subservient to an intended croonian lecture on the motion of the blood. Philosophical Transactions of the Royal Society of London 98 (1808), 164-186.

[25] ZAmir, M., AND Ritman, E. The physics of pulsatile flow. Springer, 2000. 